\title{
The adipokinetic hormones and their cognate receptor from the desert locust, Schistocerca gregaria: solution structure of endogenous peptides and models of their binding to the receptor
}

\author{
Graham Ellis Jackson ${ }^{\text {Corresp., }}$, Elumalai Pavadai ${ }^{1,2}{ }^{,}$Gerd Gäde $^{3}$, Neils Andersen ${ }^{4}$ \\ 1 Department of Chemistry, University of Cape Town, Cape Town, Western Cape, South Africa \\ 2 Department of Physiology and Biophysics, Boston University, Boston, Massachusetts, United States \\ 3 Department of Biological Sciences, University of Cape Town, Cape Town, Western Cape, South Africa \\ ${ }^{4}$ Chemistry Department, University of Washington, Seattle, Washington, United States \\ Corresponding Author: Graham Ellis Jackson \\ Email address: graham.jackson@uct.ac.za
}

Background. Neuropeptides exert their activity through binding to G-protein coupled receptors (GPCRs). GPCRs are well-known drug targets in the pharmaceutical industry and are currently discussed as targets to control pest insects. Here we investigate the neuropeptide adipokinetic hormone (AKH) system of the desert locust Schistocerca gregaria. The desert locust is known for its high reproduction, and for forming devastating swarms consisting of billions of individual insects. It is also known that $S$. gregaria produces three different AKHs as ligands but has only one AKH receptor. The AKH system is known to be essential for metabolic regulation, which is necessary for reproduction and flight activity.

Methods. Nuclear magnetic resonance techniques (NMR) in a dodecylphosphocholin (DPC) micelle solution were used to determine the structure of the three AKHs. The primary sequence of the $S$. gregaria AKH receptor (AKHR) was used to construct a 3D molecular model. Next, the 3 AKHs were individually docked to the receptor, and dynamic simulation of the whole ligand-receptor complex in a model membrane was performed.

Results. Although the three endogenous AKHs of $S$. gregaria have quite different amino acids sequences and chain length (two octa- and one decapeptide), NMR experiments assigned a turn structure in DPC micelle solution for all. The GPCR-ModSim program identified human kappa opioid receptor (hk-OR) to be the best template after which the S. gregaria AKHR was modeled. All three AKHs were found to have the same binding site on this receptor, interact with similar residues of the receptor and have comparable binding constants. Molecular switches were also identified; the movement of the receptor could be visually shown when ligands (AKHs) were docked and the receptor was activated.

Conclusions. The study proposes a model of binding of the three endogenous ligands to the one existing AKH receptor in the desert locust and paves the way to use such a model for the design of peptide analogs and finally, peptide mimetics, in the search for novel species-specific insecticides based on receptor-ligand interaction. 
1 The adipokinetic hormones and their cognate receptor

2 from the desert locust, Schistocerca gregaria:

3 solution structure of endogenous peptides and

4 models of their binding to the receptor

5

6

7

\author{
Graham E. Jackson ${ }^{1}$, Elumalai Pavadai ${ }^{1,2}$, Gerd Gäde $^{3}$ and Niels H. Andersen ${ }^{4}$
}

${ }^{1}$ Department of Chemistry, University of Cape Town, Cape Town, South Africa.

${ }^{2}$ Current address: Department of Physiology and Biophysics, Boston University School of Medicine, 700 Albany Street, Boston, MA, USA

${ }^{2}$ Department of Biological Sciences, University of Cape Town, Cape Town, South Africa

${ }^{3}$ Chemistry Department, University of Washington, Seattle, Washington, United States

Corresponding Author:

Graham E. Jackson ${ }^{1}$

Private Bag, Rondebosch, Cape Town, 7701, South Africa.

Email address: graham.jackson@uct.ac.za

\title{
Abstract
}

Background. Neuropeptides exert their activity through binding to G-protein coupled receptors (GPCRs). GPCRs are well-known drug targets in the pharmaceutical industry and are being considered as novel targets to control pest insects. Here we investigate the neuropeptide adipokinetic hormone (AKH) system of the desert locust Schistocerca gregaria. The desert locust is known for its high reproduction, and for forming devastating swarms consisting of billions of individual insects. It is also known that $S$. gregaria produces three different AKHs as ligands but has only one AKH receptor. The AKH system is known to be essential for metabolic regulation, which is necessary for reproduction and flight activity.

Methods. Nuclear magnetic resonance techniques (NMR) in a dodecylphosphocholin (DPC) micelle solution were used to determine the structure of the three AKHs. The primary sequence of the S. gregaria AKH receptor (Schgr-AKHR) was used to construct a 3D molecular model. Further, the 3 AKHs were individually docked to the receptor, and dynamic simulation of the whole ligand-receptor complex performed in a model membrane.

Results. Although the three endogenous AKHs of $S$. gregaria have quite different amino acids sequences and chain length (two octa- and one decapeptide), NMR experiments suggested that they all had a turn structure in DPC micelle solution. The human kappa opioid receptor (hк-OR) 
was identified as the best template for homology modeling of Schgr-AKHR. All three AKH peptides were found to have the same binding site on this receptor, interact with similar residues of the receptor and have comparable binding constants. Molecular switches were also identified; the movement of the receptor could be visually shown when the peptides were docked and the receptor activated.

Conclusions. The study proposes a model of binding of three endogenous ligands to the one existing AKH receptor in the desert locust and paves the way to use such a model for the design of peptide analogs and finally, peptide mimetics, in the search for novel species-specific insecticides based on receptor-ligand interaction.

\section{Introduction}

In 1976 the primary structure of the first metabolic insect neuropeptide was published (Stone et al. 1976). The decapeptide was isolated from the retrocerebral glands (corpora cardiaca) of the migratory (Locusta migratoria) and desert (Schistocerca gregaria) locusts. This neuropeptide was functionally paramount in mobilizing lipids, especially during flight episodes and, hence, was classified as an adipokinetic hormone (AKH). Its modern code name is Locmi-AKH-I (for primary structure, see Table 1). Subsequently, in both locust species, a second species-specific AKH octapeptide was found (Gäde et al. 1986; Siegert et al. 1985) (see Table 1). Later, a third $\mathrm{AKH}$, again an octapeptide, was isolated and functionally characterized from L. migratoria (Oudejans et al. 1991) (see Table 1). Genome data mining lead to the discovery of a putative fourth AKH in the migratory locust (Veenstra 2014). The sequence of this octapeptide was identical to an $\mathrm{AKH}$, Aedae-AKH, previously cloned from the yellow fever mosquito, Aedes aegypti (Kaufmann et al. 2009). It was also found in the corpora cardiaca of the alderfly, Sialis lutaria (Gäde et al. 2009) and the desert locust (Marchal et al. 2018).

All these peptides are members of the large AKH/red pigment-concentrating hormone (RPCH) family, which occurs not only in insects and crustaceans, but also evolved in molluses (Johnson et al. 2014; Li et al. 2016). The AKH gene codes an mRNA that is translated into a prepropeptide with the following features: a signal peptide is followed immediately by the respective AKH peptide, a glycine amidation site, a dibasic processing site and, C-terminally, another putative peptide of variable length (Gäde \& Marco 2013). After cleavage and posttranslational modification, the structure of the mature AKH is characterized by a chain length of 8 to 10 amino acids, a pyroglutamate residue (pGlu) at the N-terminus and a carboxyamide at the C-terminus. The amino acids Leu, Ile, Val, Tyr or Phe are found at position 2, Asn or Thr at position 3, the aromatic residues Phe or Tyr at position 4, Ser or Thr at position 5, and various amino acids at position 6,7 and 10 . The aromatic Trp is always present at position 8 and Gly at position 9 (Gäde 2004; Gäde 2009).

Most insect neuropeptides, including AKHs, exert their activity via binding to G proteincoupled receptors (GPCRs). In the pharmaceutical industry GPCRs are well known drug targets, and insect neuropeptide GPCRs are being considered as targets for the control of pest insects 
(Audsley \& Down 2015; Verlinden et al. 2014). A number of AKH receptors have been cloned and sequenced (Alves-Bezerra et al. 2016; Caers et al. 2012; Hou et al. 2017, to name a few) and the AKH system with its primarily metabolic function has been identified as a putative target as well (Gäde \& Goldsworthy 2003). With this aim, recent studies (Marchal et al. 2018) characterized the AKHR from the desert locust and used a mammalian cell-based bioluminescence assay to investigate activation of the cloned receptor by a number of naturallyoccurring peptides (Marchal et al. 2018). The first 3D-structure of an AKH hormone peptide derived from nuclear magnetic resonance (NMR) spectroscopy was published by Zubrzycki and Gäde in 1994 (Zubrzycki \& Gade 1994). Modeling of binding of members of the AKH/RPCH family to their cognate receptors has also been undertaken before: one model has been proposed for an insect, the malaria mosquito, Anopheles gambiae (Mugumbate et al. 2013) and another for a crustacean, the water flea Daphnia pulex (Jackson et al. 2018). Although the receptors share spatial regions, the binding modes of the two ligands have different orientation (Jackson et al. 2018). In both cases, however, the receptor has only one endogenous, octapeptide ligand. In the current study, three AKH neuropeptides (one decapeptide and two octapeptides, see Table 1) are present in S. gregaria; two of these peptides have been found to be active in lipid mobilization (Gäde 1990) and two of these peptides bind to the same receptor (Marchal et al. 2018). The challenge, hence, was to understand how such different peptides could all activate the same receptor. The available information on the peptides and the AKH receptor of $S$. gregaria, gives us the opportunity to model the binding of these three peptides to their cognate receptor. This information could then be used to explain how the different peptides are able to activate the same receptor. This step is necessary in order to design novel peptidomimetic agonist or antagonists, which can be synthesize and tested as cheap pest insect control substances.

\section{Materials \& Methods}

Schgr-AKH-II was synthesized by GL Biochem Ltd (Shanghai, China), Aedae-AKH by Pepmic Co.,Ltd. (Suzhou, China) and Locmi-AKH-I by Peninsula Laboratories (Belmont, California, USA). Purity was checked with HPLC-MS and it was found to be $>95$ to $98 \%$ pure. The peptides were not sufficiently soluble in water for the NMR experiments and so solutions were prepared in 30\% dimethylsulfoxide (DMSO) and/or in a dodecylphosphocholin (DPC) micelle solution. Typically, $1 \mathrm{mg}$ of sample was dissolved in $0.5 \mathrm{ml}$ of either $20 \mathrm{mM}$ phosphate buffer + $30 \%$ DMSO or 10:1 (v/v) $\mathrm{H}_{2} \mathrm{O}: \mathrm{D}_{2} \mathrm{O}$ solution which was $150 \mathrm{mM}$ in deuterated DPC-d38 (Cambridge Isotopes, $98.6 \% \mathrm{~d}$ ) and buffered with $20 \mathrm{mM}$ potassium phosphate buffer. Peptidepeptide interactions were minimized by maintaining a peptide to micelle ratio of $1: 3$, assuming 50 molecules of DPC per micelle (Jackson et al., 2018). Sodium 4,4-dimethyl-4-silapentane-1sulfonate (DSS) was used as an internal standard.

Nuclear magnetic resonance (NMR) experiments were performed on either, a Bruker Advance $700 \mathrm{MHz}$ spectrometer or a Bruker Advance $600 \mathrm{MHz}$ spectrometer with a prodigy probe. A mixing time of $60 \mathrm{~ms}$ was used for Total Correlation Spectroscopy (TOCSY) (Hwang \& Shaka 1995). A mixing time of $150 \mathrm{~ms}$ was used for nuclear Overhauser spectroscopy (NOESY) 
120 (Braunschweiler \& Ernst 1983). Spectral assignments were based on the method of Wüthrich

121 (Weber et al. 1988; Wüthrich 1986). ${ }^{13} \mathrm{C}$ assignments were based on Heteronuclear Single

122 Quantum Coherence (HSQC) spectra (Sklenar et al. 1993).

123

124

125

\section{Peptide molecular dynamics (MD)}

126

127

128

129

130

131

132

133

134

135

136

137

138

139

140

141

142

143

144

145

146

147

148

149

150

151

152

153

154

155

156

157

158

The three AKH peptides were built using Maestro [Schrödinger, Inc., New York, NY, USA] and energy minimized using a steepest descent algorithm. NMR restrained molecular dynamic simulations in vacuum, water and DPC were performed using GROMACS version 5.1.2 (Van Der Spoel et al. 2005). Although only the final results in DPC micelle solution are relevant to the current study, the three different environments were used to adequately search conformational space in a reasonable amount of time. Thus, the vacuum simulation was done at $600 \mathrm{~K}$ to allow the peptide to move faster and overcome any conformational barriers. All simulations were performed using the OPLS-AA all-atom force field with a time step of $2 \mathrm{fs}$. The LINCS algorithm was used to constraint all bonds. A cut-off of $1.0 \mathrm{~nm}$ was used for van der Waals interactions and electrostatic interactions for real space calculations. Vacuum simulations were first done to search conformational space by collecting 100 snapshots of the trajectory during a $10 \mathrm{~ns}$ simulation, at $600 \mathrm{~K}$. Each conformation was then annealed to $300 \mathrm{~K}$ over $40 \mathrm{ps}$. Cluster analysis of the resulting structures, using the linkage algorithm of GROMACS and a cut-off of $0.1 \mathrm{~nm}$ on the backbone atoms, gave one single large cluster. The conformer in the cluster with the lowest energy was used for simulations in water. Using the tip4p water model, a box containing the peptide and $\sim 7000$ water molecules was constructed. Following equilibration, molecular dynamics was performed for $10 \mathrm{~ns}$ at $300 \mathrm{~K}$ under NPT conditions. In total, 200 structures were collected at $50 \mathrm{ps}$ intervals. Cluster analysis was performed as before and the results used in the DPC/water simulations.

For simulations in a water/DPC mixture, the lowest energy structure obtained previously was placed in the center of a $7 \mathrm{~nm}$ cubic box filled with $\sim 10000$ SPC water molecules and a 52 DPC molecule micelle as obtained from Tieleman et al.(Tieleman et al. 2000) The micelle was translated so that the center of the micelle was at the bottom edge of the box. This meant that, using periodic boundary conditions, half the micelle was at the bottom of the box and the other half was at the top. The peptide was then placed in the center of the box. Energy minimization was carried out using the steepest descent method to a tolerance of $10 \mathrm{~kJ} \mathrm{~mol}^{-1}$ or to machine precision. Two stages of system equilibration were performed to solvate the peptide and to achieve a steady state starting temperature, pressure and density. The first stage of equilibration involved performing MD for $100 \mathrm{ps}$ under NVT conditions at $300 \mathrm{~K}$ followed in the second stage by a further $1 \mathrm{~ns}$ MD under NPT conditions. The final MD simulation was for $10 \mathrm{~ns}$ during which 200 snapshots were collected. Cluster analysis was performed in the same manner as before.

\section{Homology modeling}

PeerJ reviewing PDF | (2019:03:36224:1:1:NEW 9 Jul 2019) 
159 The primary sequence of the adipokinetic hormone receptor from $S$. gregaria, Schgr-AKHR, was

160

161

162

163

164

165

166

167

168

169

170

171

172

173

174

175

176

177

178

179

180

181

182

183

184

185

186

187

188

189

190

191

192

193

194

195

196

197 obtained from the GenBank (GenBank ID: AVG47955.1). Transmembrane (TM) helix predictions were computed on-line using PSIPRED (http://bioinf.cs.ucl.ac.uk/psipred/) (Jones 2007; Jones et al. 1994; Nugent \& Jones 2009). The results showed that this sequence had seven TM helices (Figure S1).

The GPCR-ModSim Web server (http:/gpcr-modsim.org/) (Rodríguez et al. 2012) was used for template selection and preliminary sequence alignment of Schgr-AKHR. This server accepts an amino acid sequence as input and searches templates by multiple sequence alignments with the

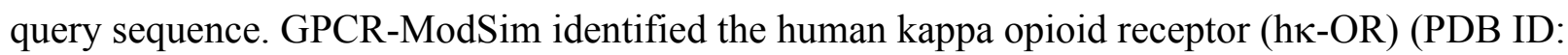
4DJH; Wu et al., 2012) as the best template. Subsequently, the sequence alignment between

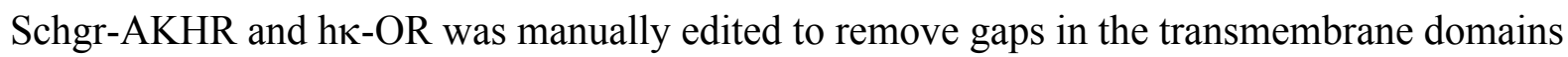
without disrupting their conserved regions. Finally, homology models for Schgr-AKHR were constructed using Modeler 9v7. This is an automated homology modeling program that performs automated protein homology modeling and loop modeling for the receptor by satisfaction of spatial restraints (Sali 1995). The quality of the selected model was evaluated for internal consistency and reliability, such as stereochemical quality, using PROCHECK (Laskowski et al. 1996). The quality of the non-bonded atom interactions was evaluated using ERRAT (Colovos \& Yeates 1993a).

\section{Docking studies}

The validated Schgr-AKHR model and the structure of the DPC micellar structure of the 3 AKH peptides were prepared for docking simulations using Protein Preparation Wizard and LigPrep of the Schrödinger suite (Schrödinger Inc., New York, NY, USA). Site-directed mutagenesis studies (Kooistra et al. 2013), molecular modeling and structural analyses (Li et al. 2010) suggest that most of the class A GPCRs share a similar binding pocket $c$.f. retinal bound to rhodopsin, carazolol bound to beta-2 adrenergic receptor (Vilar et al. 2011) and Anoga-HrTH bound to the AKH receptor of A. gambiae (Mugumbate et al. 2011). Thus, the extra-cellular portion of the receptor was used for the docking simulations.

GLIDE docking (Trott \& Olson 2010) was used for peptide docking with a grid space of $72 \times 72$ $\mathrm{x} 72$, which covered all extracellular loops and helices. The receptor grid was generated for peptide ligands and the docking precision was SP-Peptides. This setting automatically increases the number of poses collected.

\section{MD of docked structure}

The best poses from the docking studies were used as starting structures for a $2 \mu$ s molecular dynamics simulation in a 1-palmitoyl-2-oleoyl-glycero-3-phosphocholine (POPC) membrane. Using the CHARMM-GUI [www.charm-gui.org] the docked complex was placed in a POPC membrane (128 POPC molecules) such that it spanned the membrane. The construct was then converted to an OPLS-AA, all-atom, force field. Using GROMACS, 12000 water molecules were added and the charge neutralized by adding $19 \mathrm{Cl}^{-}$ions. Several steps of equilibration were

PeerJ reviewing PDF | (2019:03:36224:1:1:NEW 9 Jul 2019) 
198

199

200

201

202

203

204

205

206

207

208

209

210

211

212

213

214

215

216

217

218

219

220

221

222

223

224

225

226

227

228

229

230

231

232

233

234

235

236

used, to pack the membrane around the receptor. This was followed by $1 \mu \mathrm{s}$ of NPT simulation at $300 \mathrm{~K}$ with Berendsen pressure coupling (Berendsen et al. 1984) and a tau-p of 2.0. The free energy of binding of the final structures, from the dynamic simulations, were calculated using Prime-MM-GBSA (Schrödinger Inc., New York, NY, USA).

\section{Results}

\section{Spectral assignment}

The chemical shift assignments of the three AKH ligands in DPC are given in Tables $2-4$. Previously, Berjanskii \& Wishart (Berjanskii \& Wishart 2006) as well as Tremblay (Tremblay 2010) have shown that the chemical shifts of peptides are sensitive to the secondary structure and flexibility of the polypeptide. Structuring-induced chemical shift changes (observed shifts minus random coil reference values) were analyzed using the CSDb algorithm available at andersenlab.chem.washington.edu/CSDb/about.php (Eidenschink et al. 2009; Fesinmeyer et al. 2004). Figures $1 \mathrm{a}-\mathrm{c}$ show such plots for the three ligands. For Schgr-AKH-II both $\mathrm{H}^{\mathrm{N}}$ and $\mathrm{H}_{\alpha}$ are shifted up-field, while for Locmi-AKH-I and Aedae-AKH, only the $\mathrm{H}^{\mathrm{N}}$ deviations are upfield. The $\mathrm{H}_{\alpha}$ deviations are small and random. It has been shown that polypeptides with a helical structure have, $\mathrm{H}^{\mathrm{N}}$ and $\mathrm{H}_{\alpha}$ chemical shifts which are, on average, $-0.30 \mathrm{ppm}$ less than their random coil values, while $\beta$-sheet structures have shifts of ca. $0.6 \mathrm{ppm}$ (Szilágyi 1995). Thus, each of these peptides has a $\beta$-turn structure. Similar results were found for a number of other deca- and octapeptidic members of the AKH family, i.e. for Declu-CC, Melme-CC and Dappu-RPCH (Jackson et al. 2018). However, downfield shifts were previously found for Anoga-HrTH from the Anopheles mosquito (Mugumbate et al. 2011; Mugumbate et al. 2013). Using the Random Coil Index tool (Tremblay 2010), the chemical shifts were also used to estimate the model-free order parameter, $S^{2}$, of the peptides (see Figure 1d). An order parameter of 1 means the peptide is rigid, while an order parameter of 0 means the peptide has no structure. Figure 1d shows that Locmi-AKH-I is very ordered, with a maximum order parameter of 0.9 around proline, whereas the $\mathrm{C}$-terminal has less ordering $\left(S^{2}=0.30\right)$. On the other hand, SchgrAKH-II and Aedae-AKH are much more flexible. The order parameter for these two peptides range from 0.1 to 0.4 , which is similar to that of Dappu- $\mathrm{RPCH}$, an AKH peptide member from the crustacean water flea (Jackson et al. 2018).

\section{MD simulation with DPC micelle}

Figure 2 shows the solution structures of the 3 AKH ligands in DPC micelle solution. In each case, the molecular dynamics was started with the peptide in water, but they rapidly diffused to interact with the DPC micelle. Depending on the starting orientation of the peptide relative to the micelle, the peptide would make contact with the phospholipid and move away until a stable orientation was established. This is shown in Figure 3 where the peptide/DPC contact area is plotted as a function of time. For Locmi-AKH-I, contact between the DPC and micelle is established during the equilibration period, for Aedae-AKH it is established after $30 \mathrm{~ns}$, while for 
237 Schgr-AKH-II, even after $60 \mathrm{~ns}$ the peptide is still not permanently attached to the micelle. It is 238 interesting to note that the contact area between Locmi-AKH-I is much higher than that for the 239 other two peptides and Locmi-AKH-I is much more rigid even though it is longer, a decapeptide 240 versus an octapeptide. The interaction between the peptides and the lipid surface, as shown by 241 the contact area, is important as it has been postulated that, before the ligand binds to its receptor, 242 it first binds to the cell membrane surface. Thus, surface binding is an important step in receptor 243 activation.

244 In order to show how the structure of the peptides changed during the molecular dynamics, an 245 overlay of each peptide is shown in Figure 2a, b and c. Cluster analysis of the trajectory gave a 246 single large cluster for each peptide with a number of smaller, high energy, clusters. The root 247 conformer and an overlay of each cluster is shown in Figure 2. The predominant conformation 248 of each peptide does have a turn feature but the details differ for each AKH. Locmi-AKH-I (Fig.

249

250

251

252

253

254

255

256

257

258

259

260

261

262

263

264

265

266

267

268

269

270

271

272

273

274

275

276

$2 \mathrm{a}$ ) has a clear $\beta$-turn around its proline residue; Aedae-AKH (Fig. 2b) has a more open structure compared to the other peptides and no marked turn around proline; Schgr-AKH-II (Fig. 2c) is tightly coiled in DPC solution.

\section{Receptor Construct}

Use of the GPCR-ModSim server gave the crystal structure of the hк-OR (PDB ID: 4DJH), with $2.9 \AA$ resolution, as a top template for Schgr-AKHR. The crystal structure has the selective antagonist JDTic complexed to the receptor (Hu et al., 2012). This template has the highest sequence identity (26.33\%) compared to other templates as shown in Figure S2. hк-OR belongs to the class A (rhodopsin-like), $\gamma$-subfamily of G-protein-coupled receptors (GPCRs) and was selected to build models for Schgr-AKHR. The initial sequence alignment from the GPCRModSim server was aligned manually with the use of Chimera (Pettersen et al. 2004a; Pettersen et al. 2004b)). The predicted transmembrane helices of Schgr-AKHR and the PDB structural

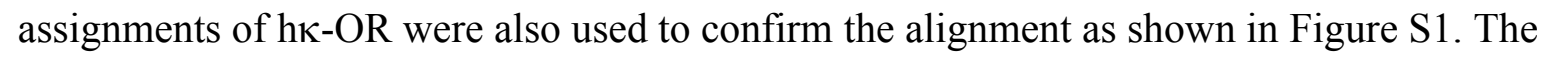
sequence analysis shows that the conserved residues in the seven TM helices (TM1-TM7) of hкOR are also highly conserved within Schgr-AKHR (conserved residues highlighted by purple coloured boxes in Figure S1), indicating that they may be involved in functions, such as

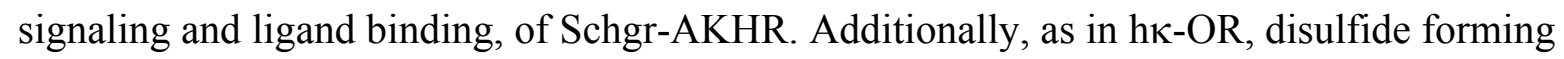
cysteine residues (Cys-131 and Cys-210) are conserved in Schgr-AKHR. The predicted TM helices of Schgr-AKHR (Figure S3) are consistent with the TM helices of hк-OR: there are no gaps or insertions in these regions, signifying that the target sequence is correctly aligned with the template sequence and, hence, can be used for the modeling process.

The homology models of Schgr-AKHR were built using the Modeler 9v7 program. The input parameters of Modeler were set to generate 100 models with high structural optimization. A disulfide bridge, Cys-131-Cys-210, was defined as in the template structure. The best model was selected based on the lowest PDF (molecular probability density function) energies and DOPE score (discrete optimized protein energy) for the docking simulations. The selected models' qualities were subsequently assessed with structural evaluation programs such as PROCHECK

PeerJ reviewing PDF | (2019:03:36224:1:1:NEW 9 Jul 2019) 
277 and ERRAT. Ramachandran plot analyses of the Schgr-AKHR model from PROCHECK are

278 shown in Figure S4. All the residues were either in favored or in allowed regions, indicating that 279 the backbone torsion angles (phi and psi) of this model are reliable. In addition, the ERRAT 280 score, so-called overall quality factor, was computed on the Schgr-AKHR model to check the 281 quality of its non-bonded atomic interactions. The normally accepted score range for a high282 quality model is $>50$ (Colovos \& Yeates 1993b). The ERRAT score for the Schgr-AKHR model 283 was 78 , showing that the model is within the high-quality range. All these validation methods 284 demonstrated that the model is reliable and can be used for further studies. The final 3D 285 structure of the Schgr-AKHR model, as shown in Figure S5A, is similar in overall fold with the 286 template protein hк-OR (Fig. S5B). The superposition of the Schgr-AKHR model with that of

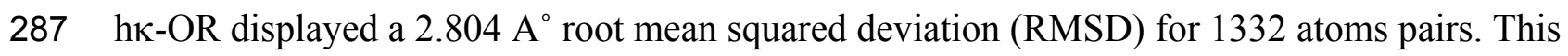
288 low RMSD demonstrates that the overall tertiary structure of the model are similar to the 289 template structure. In addition, the superimpositions indicate that the seven transmembrane 290 helices are highly conserved with hк-OR. However, there are slight structural variations in the 291 loop regions of the model.

292

293

294

295

296

297

298

299

300

301

302

303

304

305

306

307

308

309

310

311

312

313

314

315

316

\section{Ligand Docking}

LigPrep was used to generate multiple conformers of the three AKH peptides, which were then docked to the Schgr-AKHR model. A receptor grid was generated for the extra-cellular half of the GPCR and the peptide docked using SP-Peptide precision. One hundred different poses were collected and scored for each peptide. An overlay of the highest scoring poses gave the same receptor binding site for all three peptides. This binding pocket consisted of a cleft running across the top of Schgr-AKHR, between helices 2, 6 and 7 and extra-cellular loops 2 and 4 (details shown in Figures 4-6). The peptides lay along this cleft. It was found that, while the GLIDE protocol tried to dock many different conformations of the peptide, only those with some turn structure were successful. Many of the docked poses had similar GLIDE scores but different sets of peptide/receptor interactions. During the docking, it was found that the orientation of the peptide within the binding pocket did not change. For this reason, the docking was repeated with the peptide rotated through 180 degrees around the axis of the transmembrane helices i.e., the direction of $\mathrm{N}$-terminus to the $\mathrm{C}$-terminus was reversed. Again, the peptide could be docked successfully, indicating that the binding pocket was quite promiscuous. Although either orientation of the peptide would bind to the receptor, the binding energies of the two orientations differed by some $50 \mathrm{kcal} / \mathrm{mol}$ and hence the original orientation was chosen for further study.

The docked structure of each AKH peptide with the highest binding energy, was used as the starting structure for molecular dynamics of the complex in a POPC membrane. During the dynamics, the ligands were found to move, and individual side-chains rotate within the binding site, making and breaking H-bonds to various residues of the receptor. This was essentially the same as what was found during the GLIDE docking. While the POPC membrane added to the computational cost of the simulation, it was necessary to prevent the trans-membrane helices of 
317 the receptor from moving apart. Snapshots of the simulation were transferred to Maestro, where 318 Prime-MM-GBSA was used to calculate the binding energy.

319 Figure $4 \mathrm{a}$ and $\mathrm{b}$ shows an overlay of several snapshots of the dynamic simulation of the 320 octapeptide, Schgr-AKH-II, in its receptor binding pocket. As can be seen, the overall 321 conformation of Schgr-AKH-II remains the same (Figure 4c) but the side-chains move within the 322 binding site, forming and breaking intra- and inter-molecular H-bonds. There is also some

323

324

325

326

327

328

329

330

331

332

333

334

335

336

337

338

339

340

341

342

343

344

345

346

347

348

349

350

351

352

353

354

355

356 movement of the receptor during the dynamics. The free energy of binding of the different snapshots were not significantly different and ranged from -94 to $-116 \mathrm{kcal} / \mathrm{mol}$ over the $1 \mu \mathrm{s}$ simulation.

The bound conformation of Schgr-AKH-II is shown in Figure 4c, while Figure 4d is an overlay of bound Schgr-AKH-II and its lowest energy conformation in DPC micelle solution. The agreement of these two conformers is remarkable, especially considering that the GLIDE - SP protocol generates some 100 different starting conformations for the peptide docking. Figure 5a shows the bound conformation of Locmi-AKH-I, while Figure $5 \mathrm{~b}$ is an overlay of this bound conformer and the lowest energy conformer found in DPC micelle solution. The agreement here is not as close as that for Schgr-AKH-II, but the same turn structure is seen. Figure 5c shows Locmi-AKH-I in the receptor binding pocket, while Figures $5 \mathrm{~d}$ and e show the details of how Locmi-AKH-I fits into the receptor: the decapeptide stretches across the cleft in the receptor with the central portion of the peptide fitting into the binding pocket, but the two termini pointing outside the binding pocket. Locmi-AKH-I gave the most trouble during the docking stage as poses were frequently rejected. During the molecular dynamics, the terminal amide of this decapeptide was sometimes found to H-bond to a POPC molecule, which is of course not present during the GLIDE docking. The final binding energy for Locmi-AKH-I was $98 \mathrm{kcal} / \mathrm{mol}$.

Figure 6a and $\mathrm{b}$ show how Aedae-AKH fits into the binding pocket of the Schgr-AKHR model. The arrangement is similar to that of Locmi-AKH-I, except, in this case, the termini do not extend outside the receptor. In the case of Aedae-AKH, ECL4 folds over the top of the binding site, trapping the peptide inside. The conformation of bound Aedae-AKH is shown in Figure 6c, and an overlay with the DPC micelle solution conformation is shown in Figure 6d. Again, these two conformations are very similar, supporting the idea that the peptides are pre-arranged on the cell surface. The binding energy of Aedae-AKH was $-88 \mathrm{kcal} / \mathrm{mol}$.

Ligand interaction diagrams for the three ligands are shown in Figure 7, while Table 5 lists the interactions between the ligands and Schgr-AKHR. From these data, it is clear that all three AKH ligands have very similar interactions with Schgr-AKHR. Both Schgr-AKH-II and AedaeAKH, H-bond to His 169 of the receptor, while $\mathrm{W}^{8}$ of Locmi-AKH-I $\pi$-stacks with this residue. In Locmi-AKH-I, the amide carbonyl, $\mathrm{pE}^{1} \mathrm{CO}, \mathrm{H}$-bonds to Lys281, while in Aedae-AKH it is $\mathrm{pE}^{1} \mathrm{O}_{\varepsilon 1}$ which H-bonds to Lys281. The terminal residue of both Schgr-AKH-II and Locmi-AKHI H-bond to Lys288.

Analysis of molecular switches

Peerj reviewing PDF | (2019:03:36224:1:1:NEW 9 Jul 2019) 
357 A feature of class A GPCRs is the presence of highly conserved molecular switch motifs. These

358

359

360

361

362

363

364

365

366

367

368

369

370

371

372

373

374

375

376

377

378

379

380

381

382

383

384

385

386

387

388

389

390

391

392

393

394

395

396

switches, which play key roles in the stabilization of the receptor in an inactive and active state, include a TM3-6 lock, a SPLF switch, a tyrosine toggle and a DRY ionic lock. The breaking of these switches results in movement of the transmembrane helices, which can activate the receptor (Trzaskowski et al. 2012). These switches are the same as those reported for the AKH receptor of Drosphilia melanogaster, Tribolium castaneum, A. gambiae and Rhodnius prolixus (Rasmussen et al. 2015), suggesting that the activation mechanism of Schgr-AKHR may be the same.

The DRY ionic lock between arginine and tyrosine is postulated to open and close during receptor activation. This is shown in Figure 8. In the inactive state, the DRY switch is closed (Figure 8b) but upon ligand binding, TM6 and TM3 twist, opening this switch (Figure 8c). The TM3-6 lock involves two residues in the binding pocket, Arg $^{107}$ on TM3 and Tyr ${ }^{265}$ on TM6 (Figure 9a). In the inactive state these two residues are far apart, but on ligand activation, they move closer together. Fig 9a also shows, in the active state, $\operatorname{Arg}^{107} \mathrm{H}$-bonding with $\mathrm{Glu}^{190}$ of ECL4. It was noted before that this loop closes over the binding pocket after ligand binding. In Fig 9a one can also clearly see how TM6 and TM3 move together on the extra-cellular side but move away from each other on the intra-cellular side, upon activation.

The tyrosine toggle switch involves the NPxxY motif on TM7 and Tyr ${ }^{213}$ on TM5 (Fig 9b).

\section{Discussion}

As has been found before, insect neuropeptides are flexible in solution but generally have a preferred $\beta$-turn conformation (Mercurio et al. 2018; Shen et al. 2018; Zubrzycki 2000). Using CD spectroscopy, Cusinato et al. (Cusinato et al. 1998) proposed a P II extended conformation for the $\mathrm{AKH} / \mathrm{RPCH}$ peptides, at low temperatures, in aqueous solution. However, they found that the majority of AKH/RPCH peptides adopted a $\beta$-turn conformation in the presence of $0.6 \%$ SDS. This is what was found in DPC micelle solution by both NMR chemical shift and molecular modeling results for the three peptides Schgr-AKH-II, Aedae-AKH and Locmi-AKH-I in the current study. These peptides are not very soluble in water but are readily soluble in DPC micelle solution, which is a clear indication that they interact with the micelle. Previously, using DOSY NMR spectroscopy, we showed that the crustacean AKH member, Dappu-RPCH, binds to the micelle in DPC solution, and that the micelle consists of $\sim 50$ DPC molecules (Jackson et al. 2018). This was also shown by the molecular modeling for the 3 locust peptides in the present study. Locmi-AKH-I interacted strongly with the micelle and perhaps this is the reason why this peptide had a much higher order parameter than the other two peptides.

Despite chain length and sequence differences, all three peptides were found to have the same binding site on the receptor. They all had similar binding constants and interacted with the same receptor residues. This is in agreement with previous results of Marchal et al. (Marchal et al. 2018) where many members of the AKH family activated Schgr-AKHR in vitro, to the same extent and it was concluded that this receptor was quite promiscuous. In the present study, multiple binding poses were found for the peptides in the binding site and this was confirmed by

Peer) reviewing PDF | (2019:03:36224:1:1:NEW 9 Jul 2019) 
397

398

399

400

401

402

403

404

405

406

407

408

409

410

411

412

413

414

415

416

417

418

419

420

421

422

423

424

425

426

427

428

429

430

431

432

433

434

435

436

molecular dynamics in a POPC membrane. Here each peptide was found to move within the binding site, interacting with different residues. This may account for the same receptor being activated naturally by all three peptides. Interestingly, during the molecular dynamics, the receptor itself moved, closing over the binding site and opening up on the intra-cellular side. This motion has been postulated to result in receptor activation, with a G-protein able to bind to the more open receptor. Figure $8 \mathrm{a}$, which is an overlay of the active and inactive receptor, shows this movement of the helices. Measurements show that Ala-243, on TM6, moves some $6.4 \AA$ on receptor activation. One can also see that ECL6 and ECL4 (Figure 4b) close over the binding site.

It is interesting to compare and contrast the binding of Schgr-AKH-II, Aedae-AKH and LocmiAKH-I with the binding of a crustacean red pigment-concentrating hormone from Daphnia pulex, Dappu-RPCH, to its cognate receptor, Dappu-RPCHR (Jackson et al. 2018). Both receptors have similar binding sites involving TM2, 6 and 7 but Dappu-RPCHR also uses extracellular loops 1, 2 and 3, while Schgr-AKHR involves loops 2 and 4 (and loop 6 in the active receptor). Dappu-RPCH undergoes significant conformational changes upon receptor binding, having a more extended structure in solution but a more pronounced $\beta$-turn when bound. On the other hand, Schgr-AKH-II, Aedae-AKH and Locmi-AKH-I, undergo very little conformational change upon receptor binding. This might account for the higher binding constant of these three peptides relative to Dappu-RPCH. The similarity between the $\mathrm{AKH} / \mathrm{RPCH}$ systems is understandable given the evolution of the $\mathrm{AKH} /$ corazonin/ACP/GnRH receptor superfamily and their ligands. (Hauser \& Grimmelikhuijzen 2014)

The presented model of Schgr-AKHR can be compared to another class A GPCR, the human gonadotropin releasing hormone receptor, GnRHR (Flanagan \& Manilall 2017). This receptor has an exaggerated bend around Pro, in a CWxPY motif found on TM6. In GnRHR, this bend is stabilized by a water mediated H-bond between $\mathrm{Cys}^{47}$ and $\mathrm{Tyr}^{51}$ on TM6 of the CWxPY motif and by H-bonding to a residue in TM7. The presented model of Schgr-AKHR also has this CWxPY motif in TM6, which results in a proline kink. In the active receptor, Cys and Tyr residues are correctly oriented for water mediated $\mathrm{H}$-bonding, with a distance of $3.18 \AA$ between them. Also, Trp ${ }^{262}$ of the CWxPY motif was found to H-bond to $\mathrm{Asn}^{297}$ of TM7. The functional importance of this motif was demonstrated by mutations associated with congenital hypogonadotropic hypogonadism $(\mathrm{CHH})$. This rare disorder results from decreased production or secretion of gonadotropin-releasing hormone $(\mathrm{GnRH})$ and/or lack of action of GnRH upon GnRHR. There are some 25 genes identified in this condition, but if the Pro residue is substituted by Arg, there is complete disruption of the receptor function. This shows the importance of this proline kink. On the other hand, when the Cys of this receptor motif is mutated to Tyr or Ala, the GnRH ligand does not bind to the receptor (Flanagan \& Manilall 2017). Interestingly, in the inactive Schgr-AKHR, there was a H-bond between Trp-CO and Tyr-NH but there was no H-bond between $\operatorname{Trp}^{262}$ and $\mathrm{Asn}^{297}$. Upon ligand binding, however, the helices moved in such a way that the one H-bond broke and the other formed. This is similar to the DRY switch described above. Binding similarities between GnRHR and Schgr-AKHR are 
437 not unexpected. It has long been accepted that $\mathrm{GnRH}$ and $\mathrm{AKH}$ are peptides belonging to the

438 same superfamily because not only are the ligands structurally closely related but also the

439 cognate receptors (Gäde et al. 2011; Hauser \& Grimmelikhuijzen 2014; Roch et al. 2011; Roch

440 et al. 2014).

441

442 Conclusions

443 In this paper, we have shown that the putative receptor, Schgr-AKHR, is a member of the Class

444 A superfamily of G-protein coupled receptors. It has 7 transmembrane helices and the same

445 conserved residues as other AKH receptors. Schgr-AKHR also has a number of molecular

446 switch motifs, which are a feature of class A GPCRs. Our results show that the three

447 endogenous peptides, Schgr-AKH-II, Aedae-AKH and Locmi-AKH-I, all bind to the same

448 receptor binding site and with very similar binding constants. This may be surprising, as the

449 three ligands are very different; their sequence is different and one is a decapeptide, while the

450 other two are octapeptides. However, L. migratoria and S. gregaria, have only one AKH

451 receptor so it could be expected that the three endogenous peptides would have to bind to this

452 single receptor. These results also fit previous findings that, in vitro, the AKH receptor of $S$.

453 gregaria is equally well activated by a number of AKH members.

454

455 The similarities in the ligand binding of Schgr-AKHR and Dappu-RPCH/Dappu-RPCHR

456 supports the evolutionary development of the AKH/corazonin/ACP/GnRH receptor superfamily.

457 The next step in this study would be to use in silico screening to identify suitable agonists or

458 antagonists, which could act as next generation insecticides.

459

460

\section{References}

462

463 Alves-Bezerra M, De Paula IF, Medina JM, Silva-Oliveira G, Medeiros JS, Gäde G, and Gondim

464 KC. 2016. Adipokinetic hormone receptor gene identification and its role in triacylglycerol

465 metabolism in the blood-sucking insect Rhodnius prolixus. Insect Biochemistry and Molecular

466 Biology 69:51-60. https://doi.org/10.1016/j.ibmb.2015.06.013

467 Audsley N, and Down RE. 2015. G protein coupled receptors as targets for next generation

468 pesticides. Insect Biochemistry and Molecular Biology 67:27-37. S0965-1748(15)30030-8 [pii]

469 Berendsen HJC, Postma JPM, van Gunsteren WF, DiNola A, and Haak JR. 1984. Molecular

470 dynamics with coupling to an external bath. The Journal of Chemical Physics 81:3684-3690.

471 Berjanskii M, and Wishart DS. 2006. NMR: Prediction of protein flexibility. Nature Protocols 472 1:683-688. 
473 Braunschweiler L, and Ernst RR. 1983. Coherence transfer by isotropic mixing - Application to 474 proton correlation spectroscopy. Journal of Magnetic Resononance 53:521-528.

475 Caers J, Peeters L, Janssen T, De Haes W, Gäde G, and Schoofs L. 2012. Structure-activity 476 studies of Drosophila adipokinetic hormone (AKH) by a cellular expression system of dipteran 477 AKH receptors. General and Comparative Endocrinology 177:332-337.

478 10.1016/j.ygcen.2012.04.025

479 Colovos C, and Yeates TO. 1993b. Verification of protein structures: Patterns of nonbonded 480 atomic interactions. Protein Science 2:1511-1519.

481 Cusinato O, Drake AF, Gäde G, and Goldsworthy GJ. 1998. The molecular conformations of 482 representative arthropod adipokinetic peptides determined by circular dichroism spectroscopy.

483 Insect Biochemistry and Molecular Biology 28:43-50. https://doi.org/10.1016/S0965-

$484 \quad \underline{1748(97) 00094-5}$

485 Eidenschink L, Kier BL, Huggins KNL, and Andersen NH. 2009. Very short peptides with stable 486 folds: Building on the interrelationship of Trp/Trp, Trp/cation, and Trp/backbone amide 487 interaction geometries. Proteins: Structure, Function, and Bioinformatics 75:308-322.

488 10.1002/prot.22240

489 Fesinmeyer RM, Hudson FM, and Andersen NH. 2004. Enhanced hairpin stability through loop 490 design: The case of the protein G B1 domain hairpin. Journal of the American Chemical Society 491 126:7238-7243. 10.1021/ja0379520

492 Flanagan CA, and Manilall A. 2017. Gonadotropin-releasing hormone (GnRH) receptor structure 493 and GnRH binding. Frontiers in Endocrinology 8:274.

494 Gäde G. 1990. Structure-function studies on hypertrehalosaemic and adipokinetic hormones:

495 activity of naturally occurring analogues and some $\mathrm{N}$ - and C-terminal modified analogues.

496 Physiological Entomology 15:299-316. 10.1111/j.1365-3032.1990.tb00518.x

497 Gäde G. 2004. Regulation of intermediary metabolism and water balance of insects by 498 neuropeptides. Annual Review of Entomology 49:93-113.

499 Gäde G. 2009. Peptides of the adipokinetic hormone/red pigment-concentrating hormone 500 ficalamily. Annals of the New York Academy of Sciences 1163:125-136.

501 Gäde G, and Goldsworthy GJ. 2003. Insect peptide hormones: a selective review of their 502 physiology and potential application for pest control. Pest Management Science 59:1063-1075. $503 \quad 10.1002 /$ ps.755 
504 Gäde G, Goldsworthy GJ, Schaffer MH, Cook JC, and Rinehart KL. 1986. Sequence analyses of 505 adipokinetic hormones II from corpora cardiaca of Schistocerca nitans, Schistocerca gregaria, 506 and Locusta migratoria by fast atom bombardment mass spectrometry. Biochemical and 507 Biophysical Research Communications 134:723-730.

508 Gäde G, and Marco HG. 2013. Chapter 28 - AKH/RPCH Peptides. In: Kastin AJ, ed. Handbook 509 of Biologically Active Peptides (Second Edition). Boston: Academic Press, 185-190.

510 Gäde G, Šimek P, and Marco HG. 2009. The first identified neuropeptide in the insect order 511 Megaloptera: A novel member of the adipokinetic hormone family in the alderfly Sialis lutaria.

512 Peptides 30:477-482.

513 Gäde G, Šimek P, and Marco HG. 2011. An invertebrate [hydroxyproline]-modified

514 neuropeptide: Further evidence for a close evolutionary relationship between insect adipokinetic

515 hormone and mammalian gonadotropin hormone family. Biochemical and Biophysical Research

516 Communications 414:592-597. https://doi.org/10.1016/j.bbrc.2011.09.127

517 Hauser F, and Grimmelikhuijzen CJP. 2014. Evolution of the AKH/corazonin/ACP/GnRH

518 receptor superfamily and their ligands in the Protostomia. General and Comparative

519 Endocrinology 209:35-49. 10.1016/j.ygcen.2014.07.009

520 Hou Q-L, Chen E-H, Jiang H-B, Wei D-D, Gui S-H, Wang J-J, and Smagghe G. 2017.

521 Adipokinetic hormone receptor gene identification and its role in triacylglycerol mobilization

522 and sexual behavior in the oriental fruit fly (Bactrocera dorsalis). Insect Biochemistry and

523 Molecular Biology 90:1-13. https://doi.org/10.1016/j.ibmb.2017.09.006

524 Hwang TL, and Shaka AJ. 1995. Water suppression that works. Excitation sculpting using

525 arbitrary wave-forms and pulsed-field gradients. Journal of Magnetic Resonance, Series A 526 112:275-279. https://doi.org/10.1006/jmra.1995.1047

527 Jackson GE, Pavadai E, Gäde G, Timol Z, and Andersen NH. 2018. Interaction of the red 528 pigment-concentrating hormone of the crustacean Daphnia pulex, with its cognate receptor, 529 Dappu-RPCHR: A nuclear magnetic resonance and modeling study. International Journal of 530 Biological Macromolecules 106:969-978. https://doi.org/10.1016/j.ijbiomac.2017.08.103

531 Johnson JI, Kavanaugh SI, Nguyen C, and Tsai P-S. 2014. Localization and functional 532 characterization of a novel adipokinetic hormone in the mollusk, Aplysia californica. PLOS One 533 9:e106014. 10.1371/journal.pone.0106014

534 Jones DT. 2007. Improving the accuracy of transmembrane protein topology prediction using 535 evolutionary information. Bioinformatics 23:538-544. 
536 Jones DT, Taylor WR, and Thorton JM. 1994. A model recognition approach to the prediction of 537 all-helical membrane-protein structure and topology. Biochemistry 33:3038-3049.

538 Kaufmann C, Merzendorfer H, and Gäde G. 2009. The adipokinetic hormone system in 539 Culicinae (Diptera: Culicidae): Molecular identification and characterization of two adipokinetic 540 hormone (AKH) precursors from Aedes aegypti and Culex pipiens and two putative AKH 541 receptor variants from A. aegypti. Insect Biochemistry and Molecular Biology 39:770-781.

542 Kooistra AJ, Kuhne S, De Esch IJP, Leurs R, and De Graaf C. 2013. A structural 543 chemogenomics analysis of aminergic GPCRs: Lessons for histamine receptor ligand design.

544 British Journal of Pharmacology 170:101-126. 10.1111/bph.12248

545 Laskowski RA, Rullmann JAC, MacArthur MW, Kaptein R, and Thornton JM. 1996. AQUA 546 and PROCHECK-NMR: Programs for checking the quality of protein structures solved by NMR. 547 Journal of Biomolecular NMR 8:477-486.

548 Li S, Hauser F, Skadborg SK, Nielsen SV, Kirketerp-Møller N, and Grimmelikhuijzen CJP. 549 2016. Adipokinetic hormones and their G protein-coupled receptors emerged in Lophotrochozoa. 550 Scientific Reports 6:32789. 10.1038/srep32789 https://www.nature.com/articles/srep32789 551 supplementary-information

552 Li YY, Hou TJ, and Goddard III WA. 2010. Computational modeling of structure-function of G 553 protein-coupled receptors with applications for drug design. Current Medicinal Chemistry 554 17:1167-1180. 10.2174/092986710790827807

555 Marchal E, Schellens S, Monjon E, Bruyninckx E, Marco H.G., Gäde G, Vanden Broeck J, and 556 Verlinden H. 2018. Analysis of peptide ligand specificity of different insect adipokinetic

557 hormone receptors. International Journal of Molecular Science 19(2) pii E542 doi:

558 10.3390/ijms19020542.

559 Mercurio FA, Scaloni A, Caira S, and Leone M. 2018. The antimicrobial peptides casocidins I 560 and II: Solution structural studies in water and different membrane-mimetic environments.

561 Peptides. https://doi.org/10.1016/j.peptides.2018.09.004

562 Mugumbate G, Jackson GE, and van der Spoel D. 2011. Open conformation of adipokinetic 563 hormone receptor from the malaria mosquito facilitates hormone binding. Peptides 32:553-559. 564 10.1016/j.peptides.2010.08.017

565 Mugumbate G, Jackson GE, van der Spoel D, Koever KE, and Szilagyi L. 2013. Anopheles 566 gambiae, Anoga-HrTH hormone, free and bound structure - A nuclear magnetic resonance 567 experiment. Peptides 41:94-100. 10.1016/j.peptides.2013.01.008 
568 Nugent T, and Jones DT. 2009. Transmembrane protein topology prediction using support vector 569 machines. BMC Bioinformatics 10. 10.1186/1471-2105-10-159

570 Oudejans RCHM, Kooiman FP, Heerma W, Versluis C, Slotboom AJ, and Beenakkers AMT. 571 1991. Isolation and structure elucidation of a novel adipokinetic hormone (Lom-AKH-III) from 572 the glandular lobes of the corpus cardiacum of the migratory locust, Locusta migratoria.

573 European Journal of Biochemistry 195:351-359.

574 Pettersen EF, Goddard TD, Huang CC, Couch GS, Greenblatt DM, Meng EC, and Ferrin TE. 575 2004a. UCSF Chimera - A visualization system for exploratory research and analysis. Journal of 576 Computational Chemistry 25:1605-1612. 10.1002/jcc.20084

577 Rasmussen M, Leander M, Ons S, and Nichols R. 2015. Conserved molecular switch interactions 578 in modeled cardioactive RF-NH2 peptide receptors: Ligand binding and activation. Peptides 579 71:259-267. 10.1016/j.peptides.2015.07.012

580 Roch GJ, Busby ER, and Sherwood NM. 2011. Evolution of GnRH: Diving deeper. General and 581 Comparative Endocrinology 171:1-16. https://doi.org/10.1016/j.ygcen.2010.12.014

582 Roch GJ, Busby ER, and Sherwood NM. 2014. GnRH receptors and peptides: Skating backward. 583 General and Comparative Endocrinology 209:118-134.

584 https://doi.org/10.1016/j.ygcen.2014.07.025

585 Rodríguez D, Bello X, and Gutiérrez-de-Terán H. 2012. Molecular modelling of G protein586 coupled receptors through the web. Molecular Informatics 31:334-341. 10.1002/minf.201100162

587 Sali A. 1995. Comparative protein modeling by satisfaction of spatial restraints. Molecular 588 Medicine Today 1:270-277.

589 Shen Z, Jiang X, Yan L, Chen Y, Wang W, Shi Y, Shi L, Liu D, and Zhou N. 2018. Structural 590 basis for the interaction of diapause hormone with its receptor in the silkworm, Bombyx mori. 591 The FASEB Journal 32:1338-1353. 10.1096/fj.201700931R

592 Siegert K, Morgan P, and Mordue W. 1985. Primary structures of locust adipokinetic hormones 593 II. . Biological Chemistry Hoppe-Seyler 366:723-727.

594 Sklenar V, Piotto M, Leppik R, and Saudek V. 1993. Gradient-tailored water suppression for 1H595 15N HSQC experiments optimized to retain full sensitivity. Journal of Magnetic Resononance A 596 102:241-245.

597 Stone JV, Mordue W, Batley KE, and Morris HR. 1976. Structure of locust adipokinetic 598 hormone, a neurohormone that regulates lipid utilisation during flight. Nature 263:207-211.

$59910.1038 / 263207 \mathrm{a} 0$ 
600 Szilágyi L. 1995. Chemical shifts in proteins come of age. Progress in Nuclear Magnetic

601 Resonance Spectroscopy 27:325-442. 10.1016/0079-6565(95)01011-2

602

603

604 Tieleman DP, Van Der Spoel D, and Berendsen HJC. 2000. Molecular dynamics simulations of 605 dodecylphosphocholine micelles at three different aggregate sizes: Micellar structure and chain 606 relaxation. Journal of Physical Chemistry B 104:6380-6388.

607 Tremblay M-L. 2010. The predictive accuracy of secondary chemical shifts is more affected by 608 protein secondary structure than solvent environment. Journal of Biomolecular NMR 46:257.

609 10.1007/s10858-010-9400-5

610 Trott O, and Olson AJ. 2010. Software news and update AutoDock Vina: Improving the speed 611 and accuracy of docking with a new scoring function, efficient optimization, and multithreading. 612 Journal of Computational Chemistry 31:455-461. 10.1002/jcc.21334

613 Trzaskowski B, Latek D, Yuan S, Ghoshdastider U, Debinski A, and Filipek S. 2012. Action of 614 molecular switches in GPCRs - Theoretical and experimental studies. Current Medicinal 615 Chemistry 19:1090-1109. 10.2174/092986712799320556

616 Van Der Spoel D, Lindahl E, Hess B, Groenhof G, Mark AE, and Berendsen HJC. 2005.

617 GROMACS: Fast, flexible, and free. Journal of Computational Chemistry 26:1701-1718.

618 Veenstra JA. 2014. The contribution of the genomes of a termite and a locust to our

619 understanding of insect neuropeptides and neurohormones. Frontiers in Physiology 5:454.

620 Verlinden H, Vleugels R, Zels S, Dillen S, Lenaerts C, Crabbé K, Spit J, and Vanden Broeck J. 621 2014. Receptors for neuronal or endocrine signalling molecules as potential targets for the 622 control of insect pests. Advances in Insect Physiology 46: 167-303.

623 Vilar S, Ferino G, Phatak SS, Berk B, Cavasotto CN, and Costanzi S. 2011. Docking-based 624 virtual screening for ligands of $G$ protein-coupled receptors: Not only crystal structures but also 625 in silico models. Journal of Molecular Graphics and Modelling 29:614-623.

626 10.1016/j.jmgm.2010.11.005

627 Weber PL, Morrison R, and Hare D. 1988. Determining stereo-specific 1H Nuclear magnetic 628 resonance assignments from distance geometry calculations. Journal of Molececular Bioliology 629 204:483-487.

630 Wüthrich K. 1986. NMR of Proteins and Nucleic Acids. New York: Wiley.

631 Zubrzycki IZ. 2000. Molecular dynamics study on an adipokinetic hormone peptide in aqueous 632 solution. Zeitschrift für Naturforschung C, A Journal of Biosciences 55:125-128. 
633 Zubrzycki IZ, and Gäde G. 1994. Conformational study on an insect neuropeptide of the 634 AKH/RPCH-family by combined 1H-NMR spectroscopy and molecular mechanics. Biochemical 635 and Biophysical Research Communications 198:228-235.

636 https://doi.org/10.1006/bbrc.1994.1032

637

638

639 
Figure 1

NMR chemical shift deviations and model-free order parameters of Schgr-AKH-II, LocmiAKH-I and Aedae-AKH in DPC micelle solution.

Plots of $\mathrm{H}_{\alpha}$ and $\mathrm{H}^{\mathrm{N}}$ random coil chemical shift deviations of: (a) Schgr-AKH-II. (b) Locmi-AKH-I (c). Aedae-AKH. (d) Model-free order parameter, $S^{2}$.

A

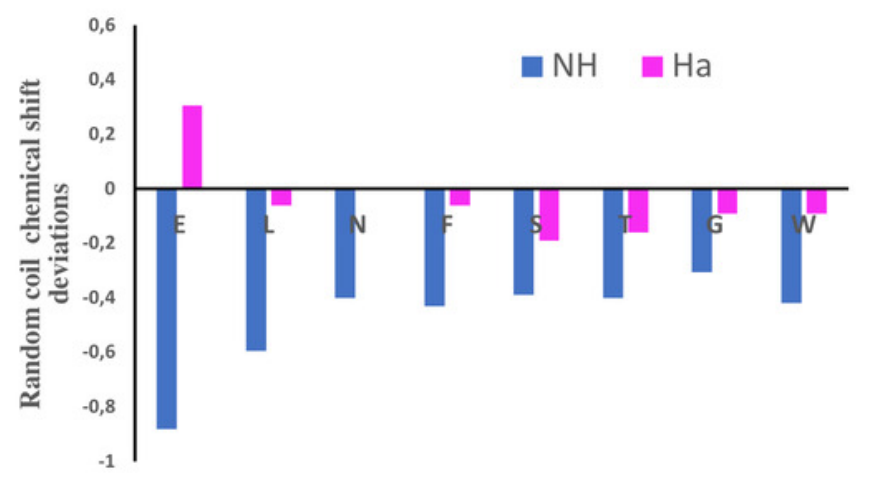

B

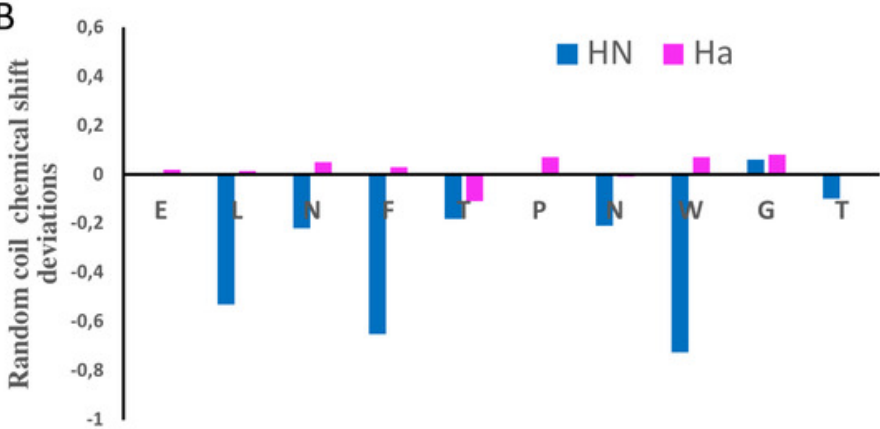

C

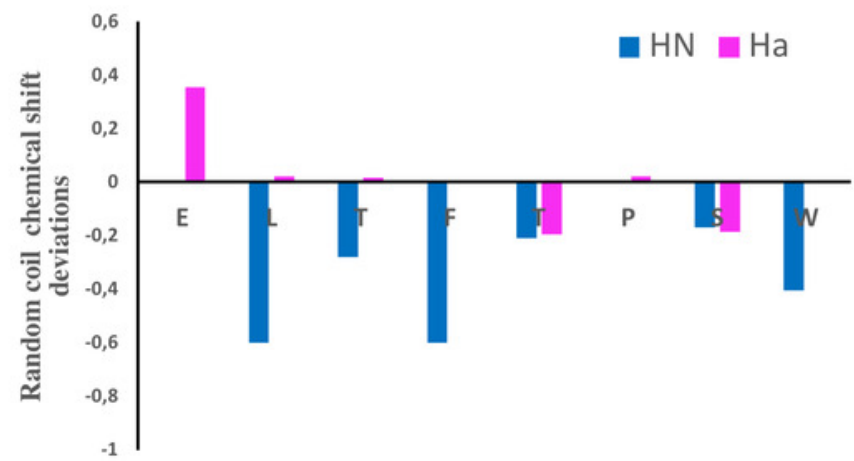

D

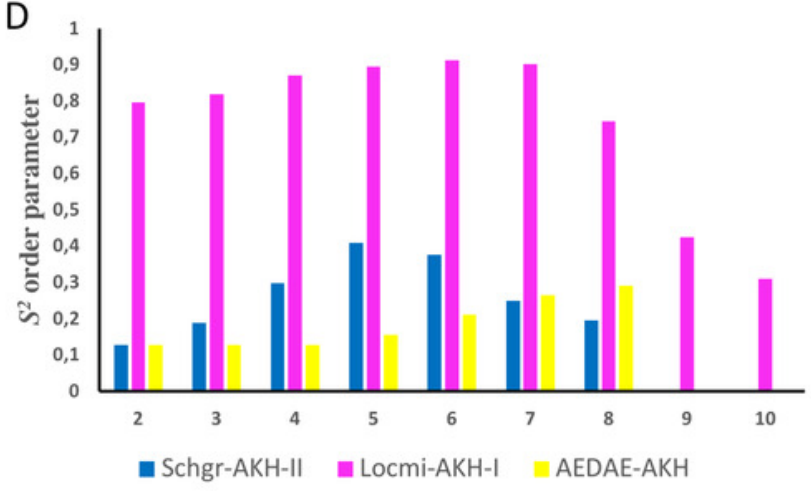


Figure 2

Solution cluster overlay and the root conformation of Schgr-AKH-II, Locmi-AKH-I and Aedae-AKH in DPC micelle solution.

(A) Cluster overlay of Locmi-AKH-I. (B) Root conformer of Locmi-AKH-I. (C) Cluster overlay of Aedae-AKH-I. (D) Root conformer of Aedae-AKH-I. (E) Cluster overlay of Schgr-AKH-II. (F) Root conformer of Schgr-AKH-II 

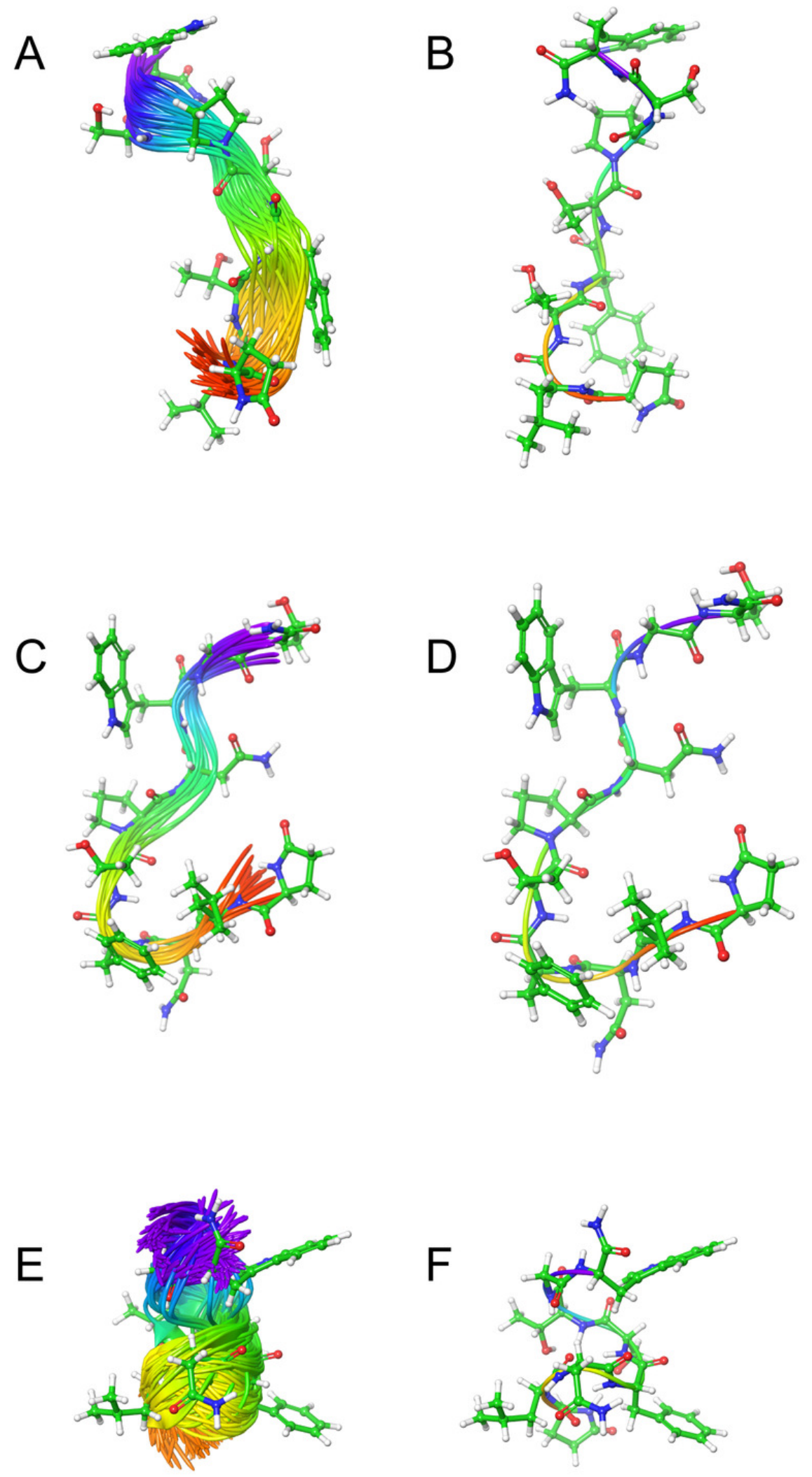
Figure 3

Contact surface area between the peptides and DPC micelle.

Plot of contact area between peptide and a DPC micelle during a 60 ps simulation of LocmiAKH-I, Aedae-AKH-I and Schgr-AKH-II, as a function of time.

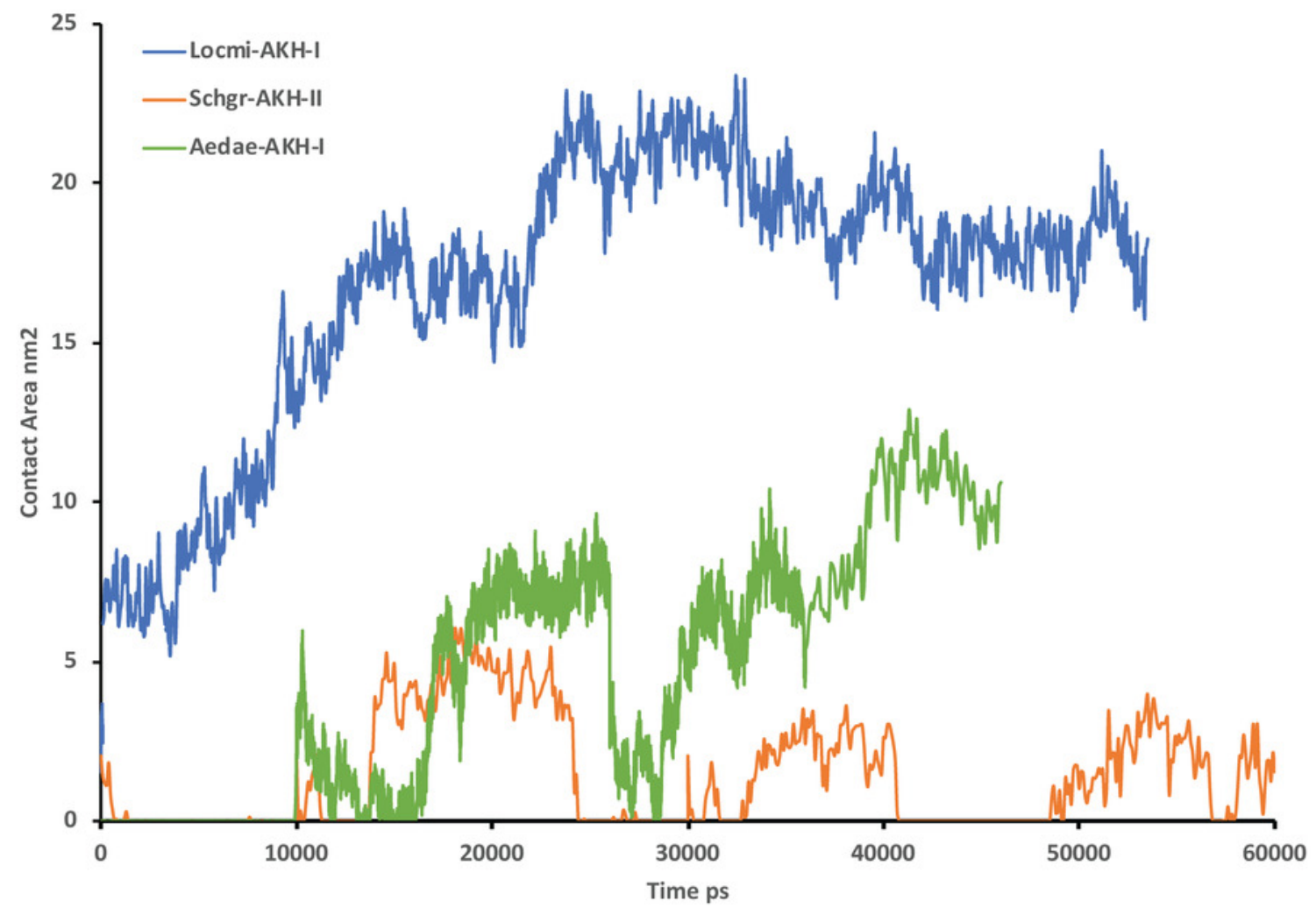




\section{Figure 4}

Structure of Schgr-AKH-II in solution and bound to Schgr-AKHR.

(a) Overlay of several snapshots of the dynamic simulation of Schgr-AKH-II in the Schgr-AKH Rreceptor binding pocket. Helices are labelled $\mathrm{H} 1$ - 7. (b) Enlargement of binding pocket showing orientation of peptide. Important extracellular loops are labelled. (c) Conformation of bound Schgr-AKH-II. (d) Overlay of best binding pose and root conformer from simulation in DPC micelle solution. 

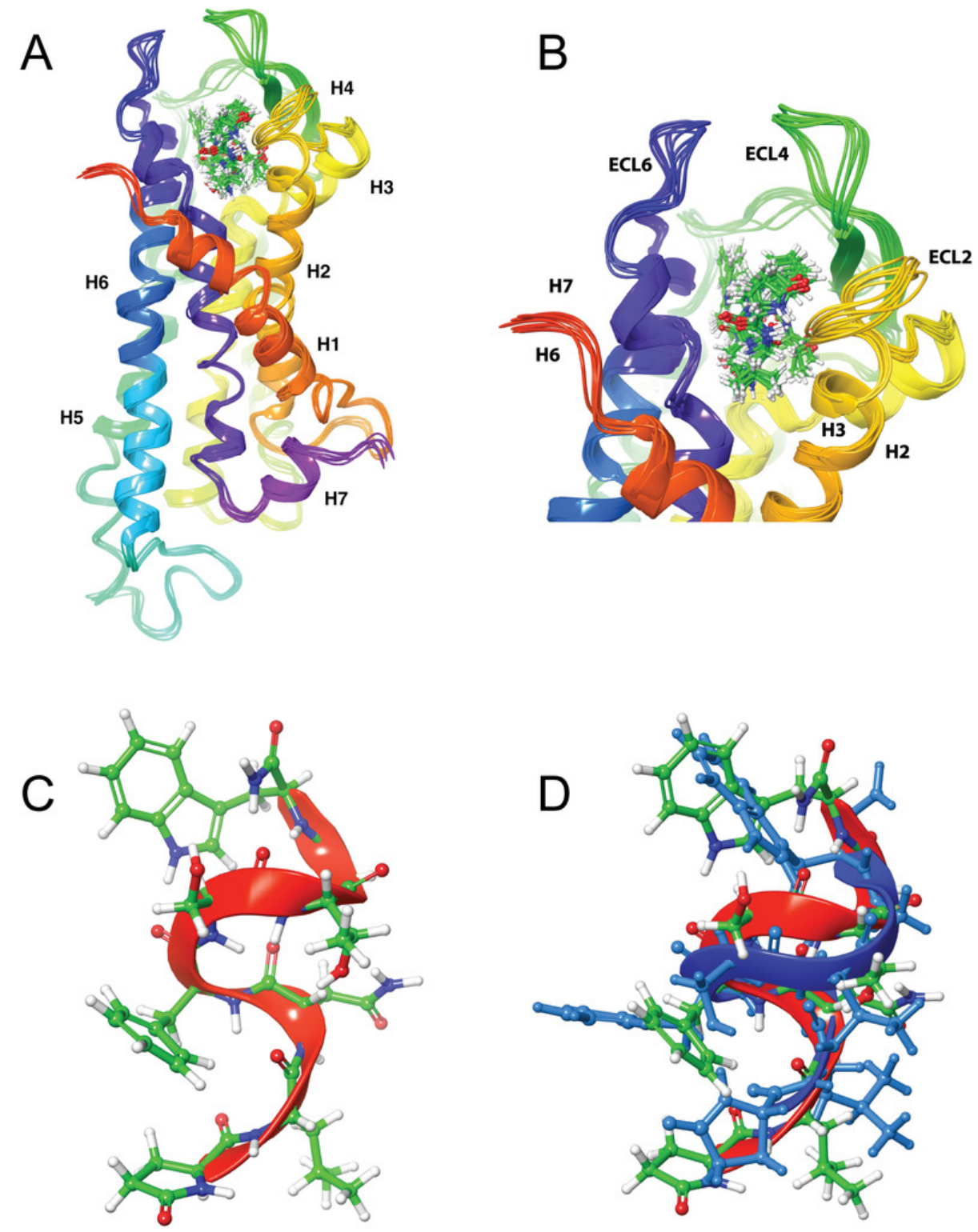


\section{Figure 5}

Structure of Locmi-AKH-I in solution and bound to Schgr-AKHR.

(a) Conformer of bound Locmi-AKH-I. (b) overlay of bound Locmi-AKH-I (blue) and in DPC micelle solution. (c) Pictorial representation of Schgr-AKHR with Locmi-AKH-I in the binding pocket. (d) Enlargement of binding pocket showing the orientation of the peptide. (e) Binding pocket surface with Locmi-AKH-I inserted. Surface is coloured according to electrostatic potential, red for negative potential, blue for positive potential and grey for neutral potential. 

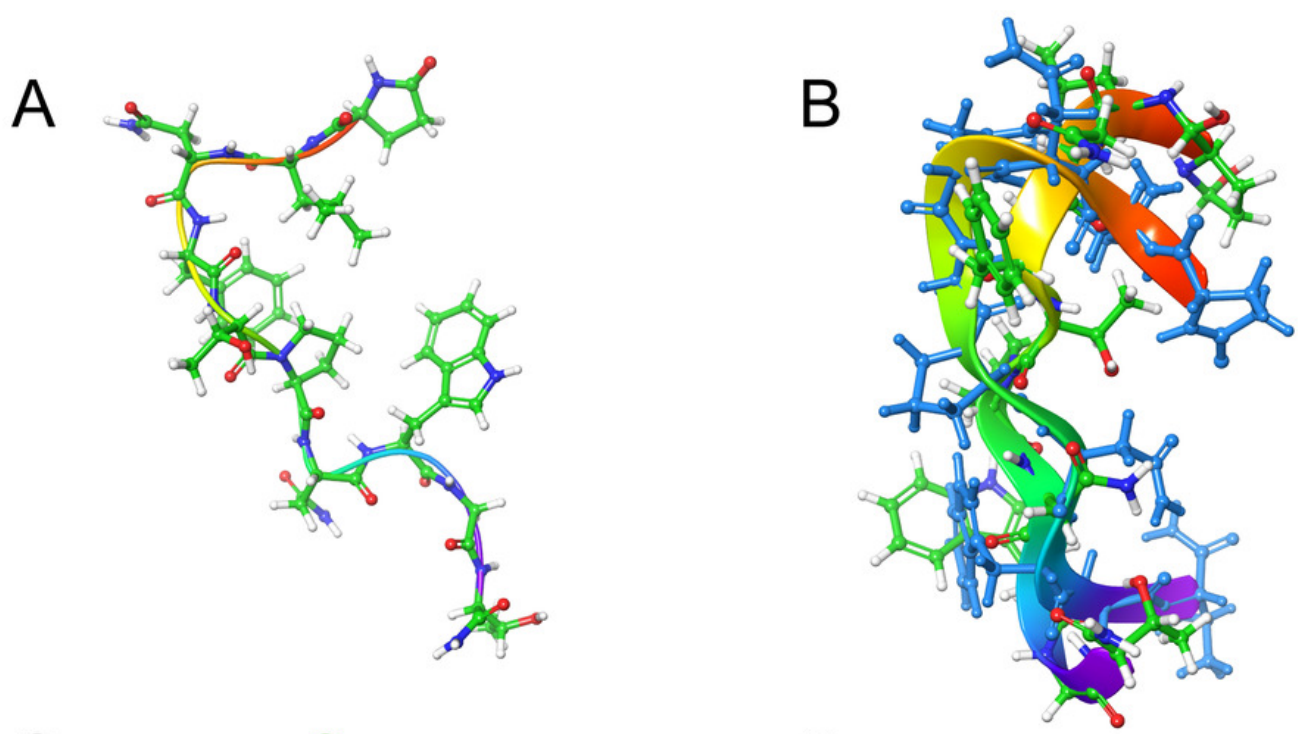

C
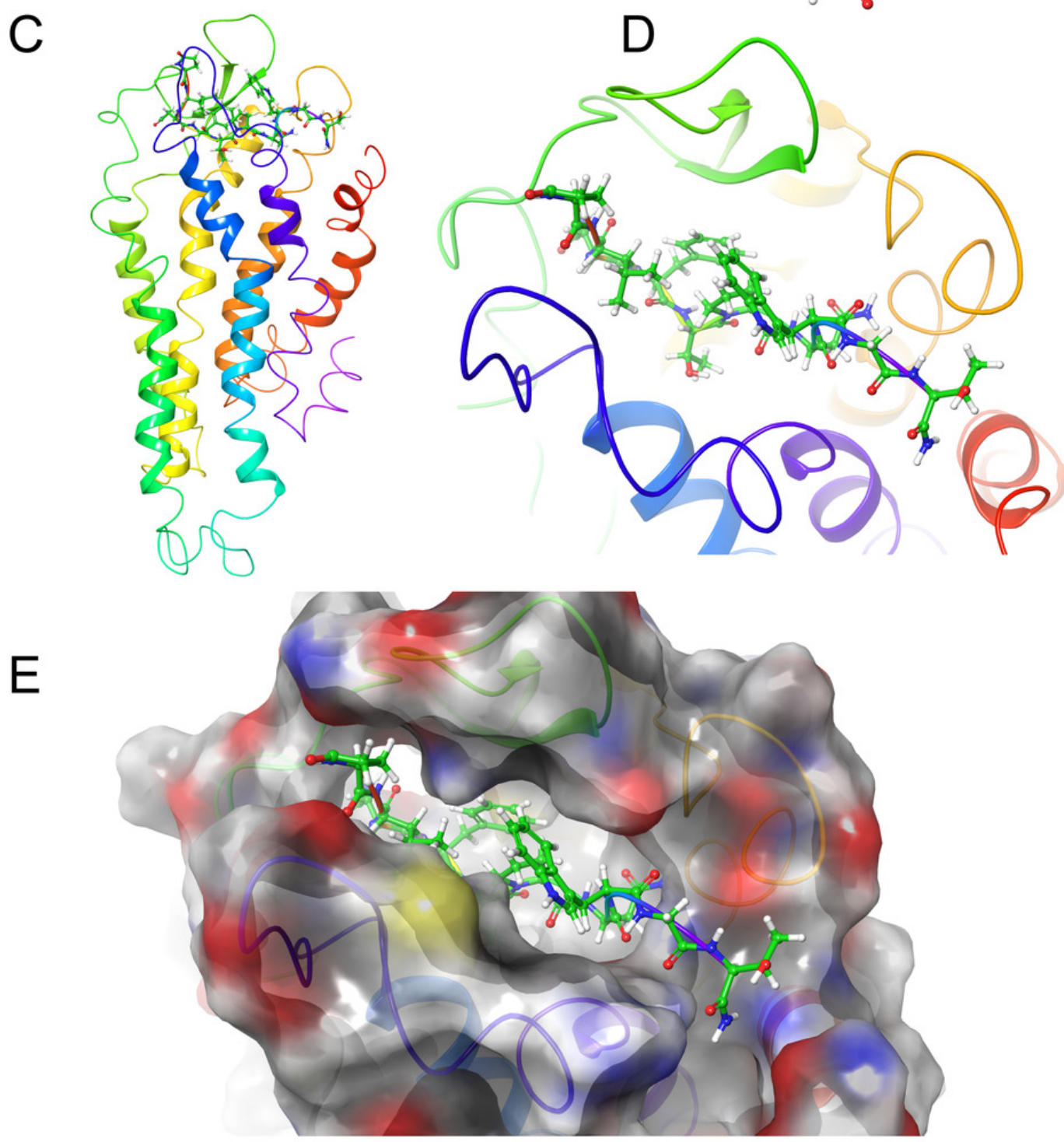


\section{Figure 6}

Structure of Aedae-AKH in solution and bound to Schgr-AKHR.

(a) Pictorial representation of Schgr-AKHR with Aedae-AKH in the binding pocket. (b) Enlargement of binding pocket showing the orientation of peptide. (c) Conformer of bound Aedae-AKH. (d) Overlay showing the different conformers of bound Aedae-AKH (blue) and in DPC solution. (e) Binding pocket surface with Aedae-AKH inserted. Surface colored according to electrostatic potential, red for negative potential, blue for positive potential and green for neutral potential. 

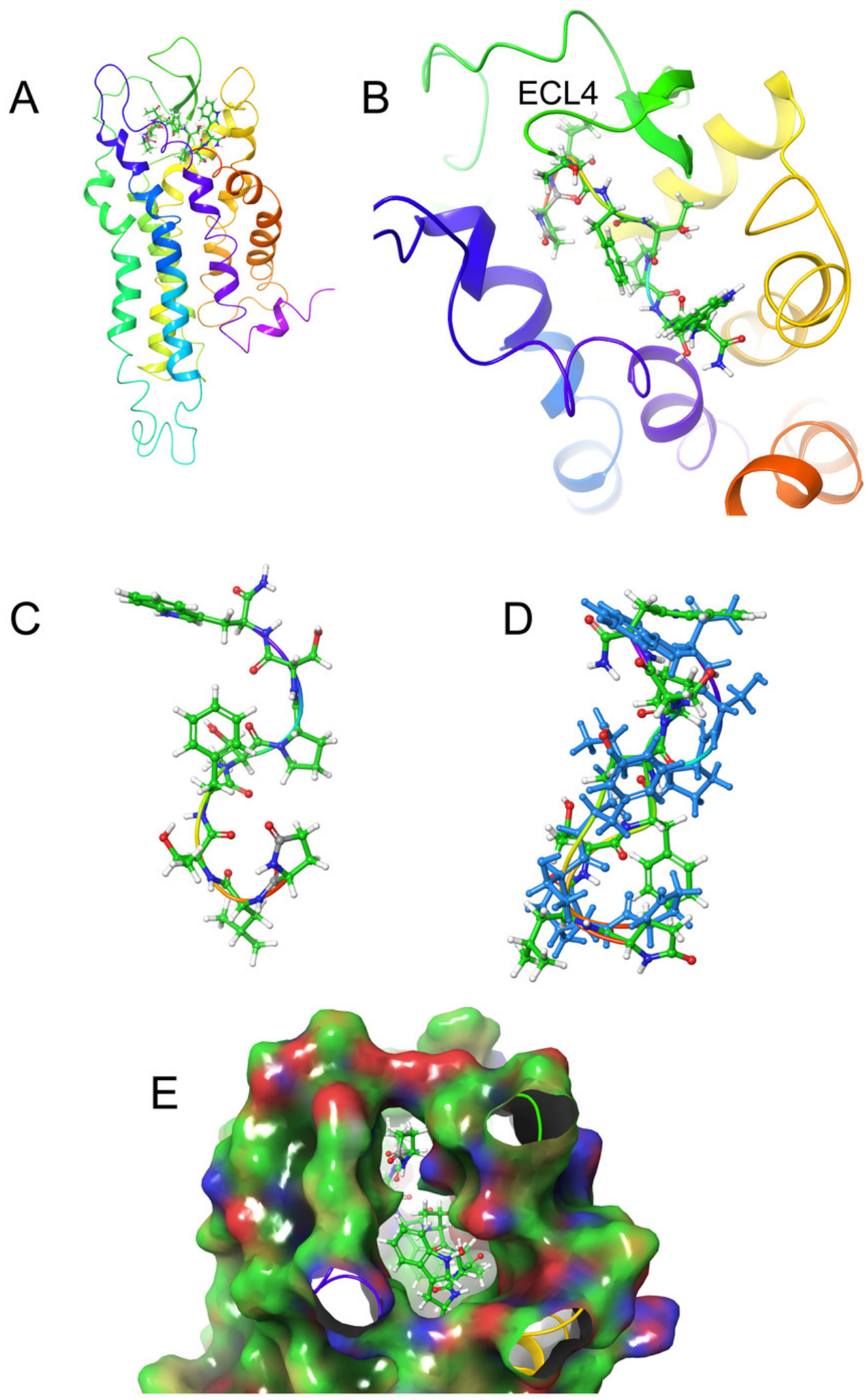


\section{Figure 7}

Protein - Ligand interaction diagrams.

(a) Aedae-AKH, (b) Schgr-AKH-II, (c) Locmi-AKH-I. Residues are represented as colored spheres, labeled with the residue name and residue number, and colored according to their properties, green for hydrophobic, blue for positive charge and red for negative charge. The ligand is displayed as a 2D structure. Interactions between the residues and the ligand are drawn as lines, colored by interaction type, purple for $\mathrm{H}$-bonding and green for pi-pi stacking. The binding pocket is indicated by a line drawn around the ligand, colored by the color of the nearest residue. Solvent exposure is indicated on the ligand atoms, and by the break in the line drawn around the pocket. 

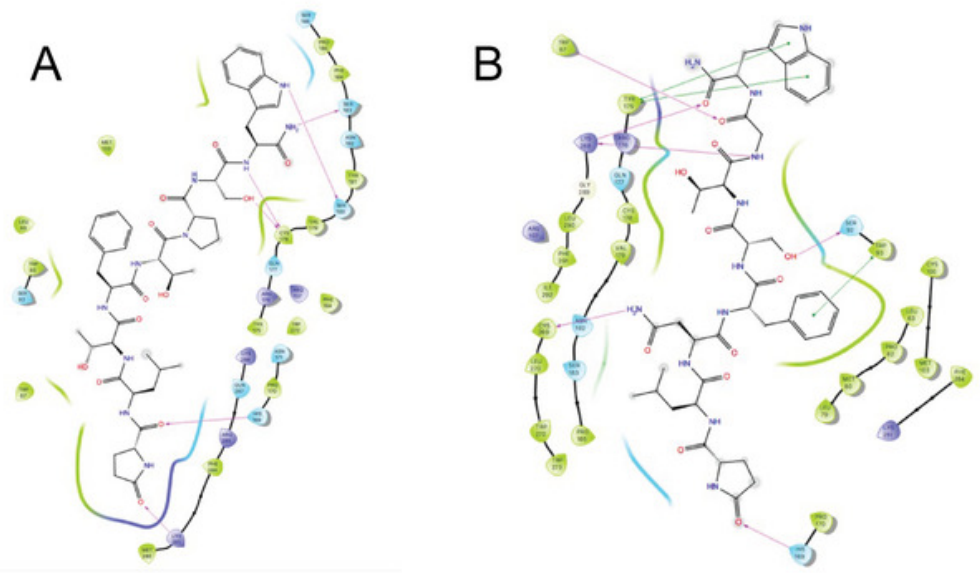

C

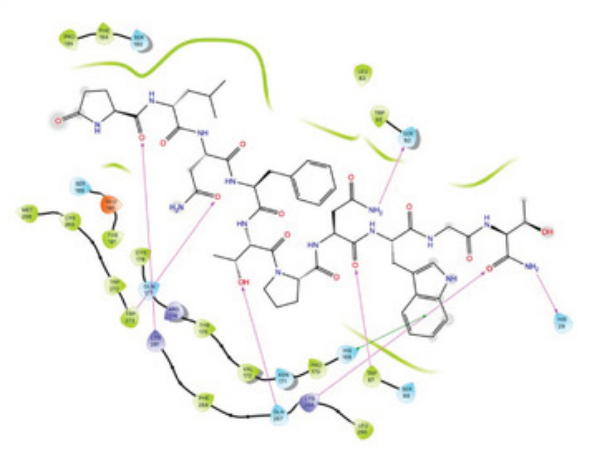


Figure 8

Comparison of active and inactive Schgr-AKH-II receptor.

(a) Overlay of active (colored) and inactive (green) S. gregaria receptor. (b) Closed DRY switch. (c) open DRY switch. Only polar hydrogens are shown. 
Peer

Manuscript to be reviewed
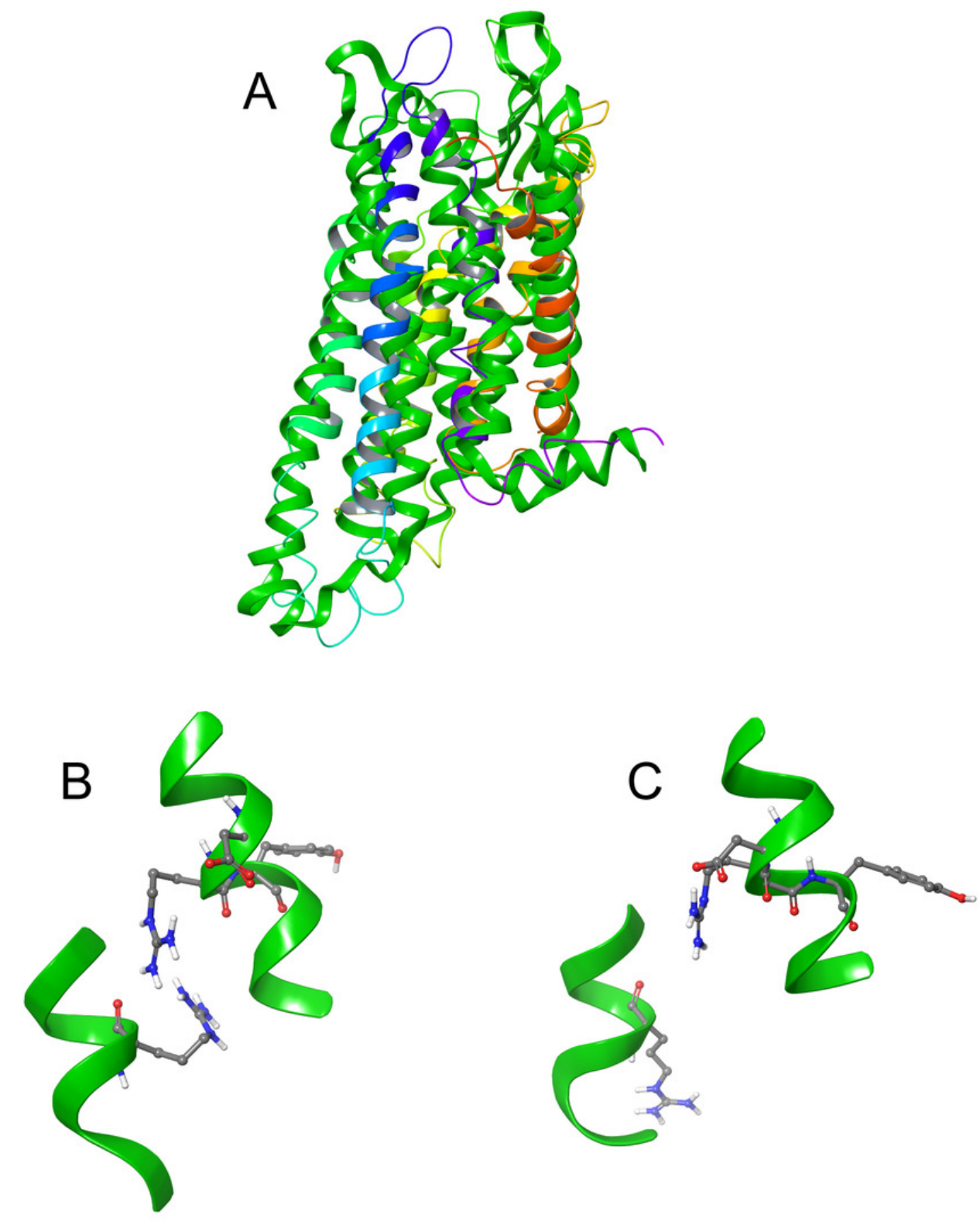

Peer j reviewing PDF | (2019:03:36224:1:1:NEW 9 Jul 2019) 
Figure 9

Schgr-AKHR molecular switches of the active and inactive receptor.

(A) TM3-6 lock of inactive receptor. (B) TM3-6 lock of active receptor. (C) Tyrosine toggle switch of inactive receptor. (D) Tyrosine toggle switch of active receptor. (E) CWXPY motif on TM6 of inactive receptor. (F) CWXPY motif on TM6 of active receptor. 

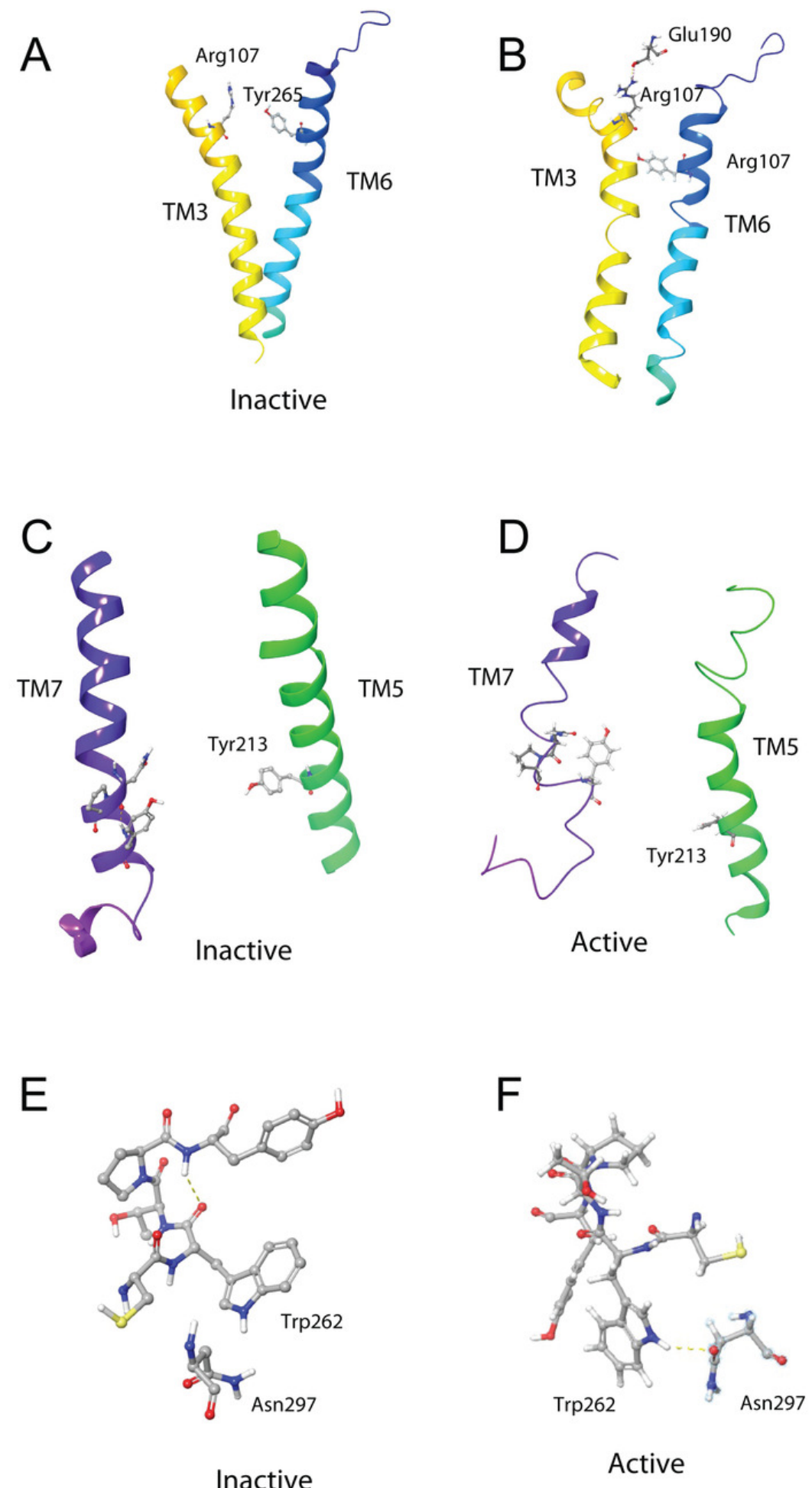


\section{Table 1 (on next page)}

Primary structure of the adipokinetic peptides of locusts. Conserved residues are highlighted in bold and underlined. 
1 Table 1. Primary structure of the adipokinetic peptides of locusts. Conserved residues are

2 highlighted in bold and underlined.

3 Peptide name

4 Locmi-AKH-I

5 Locmi-AKH-II

6 Locmi-AKH-III

7 Locmi-AKH-IV

8 (= Aedae-AKH)

9 Schgr-AKH-II
Sequence

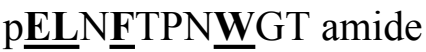

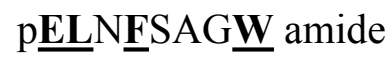

pELNETPW $\underline{\mathbf{E}}$ amide

pELTETPSW amide

pELNESTG $\underline{\mathbf{E}}$ amide

\section{Species}

L. migratoria; $S$. gregaria

L. migratoria

L. migratoria

L. migratoria, S. gregaria

S. gregaria

10

11 


\section{Table 2 (on next page)}

${ }^{1} \mathrm{H}$ chemical shifts of Schgr-AKH-II.

A. A. DPC micelle solution, $\mathrm{pH} 4.5,20 \mathrm{mM}$ phosphate, Temp $=280 \mathrm{~K}$

B. $30 \%$ DMSO solution, $\mathrm{pH} 4.5,20 \mathrm{mM}$ phosphate, Temp $=298 \mathrm{~K}$ 
1 Table 2. $1 \mathrm{H}$ chemical shifts of Schgr-AKH-II:

2 A. DPC micelle solution, $\mathrm{pH} 4.5,20 \mathrm{mM}$ phosphate, $\mathrm{Temp}=280 \mathrm{~K}$

$\begin{array}{llllll}\text { \# } & \text { Res } & \mathbf{H N} & \mathbf{H} \boldsymbol{\alpha} & \mathbf{H \beta}\left(\mathbf{H} \boldsymbol{\beta}^{\prime}\right) & \text { Others } \\ 1 & \text { Glu } & 7.742 & 4.361 & 2.335, & \mathrm{H} \gamma: 2.483,1.951 \\ 2 & \text { Leu } & 8.259 & 4.232 & 1.614,1.318 & \mathrm{H} \delta: 0.863 \\ 3 & \text { Asn } & 8.364 & 4.660 & 2.767,2.647 & \mathrm{H} \delta: 6.864,7.565 \\ 4 & \text { Phe } & 7.978 & 4.561 & 3.071,2.992 & \\ 5 & \text { Ser } & 8.096 & 4.289 & 3.782,3.689 & \\ 6 & \text { Thr } & 7.923 & 4.179 & 3.908 & \mathrm{H} \gamma: 1.040 \\ 7 & \text { Gly } & 8.145 & 3.845,3.905 & & \\ 8 & \text { Trp } & 7.790 & 4.587 & 3.181,3.183 & \mathrm{H} \delta: 7.164, \mathrm{H} \varepsilon: 9.894,7.513, \\ \end{array}$

B. $30 \%$ DMSO solution, $\mathrm{pH} 4.5,20 \mathrm{mM}$ phosphate, Temp $=298 \mathrm{~K}$

\begin{tabular}{|c|c|c|c|c|c|}
\hline$\#$ & Res & HN & $\mathbf{H \alpha}$ & $\mathbf{H \beta}\left(\mathbf{H}^{\prime}\right)$ & Others \\
\hline 1 & Glu & 7.770 & 4.173 & 2.238 & $\mathrm{H} \gamma: 2.361,1.888$ \\
\hline 2 & Leu & 8.199 & 4.160 & $1.436,1.304$ & $\mathrm{H} \delta: 0.732,0.780$ \\
\hline 3 & Asn & 8.208 & 4.501 & $2.519,2.594$ & H $\delta: 6.777,7.440$ \\
\hline 4 & Phe & 8.077 & 4.467 & $3.046,2.849$ & \\
\hline 5 & Ser & 8.134 & 4.318 & $3.710,3.667$ & \\
\hline 6 & Thr & 7.917 & 4.165 & 4.076 & $\mathrm{H} \gamma: \mathrm{na}, 1.050$ \\
\hline 7 & Gly & 8.132 & $3.794,3.691$ & & \\
\hline & Trp & 7.874 & 4.466 & $3.187,3.018$ & $\begin{array}{l}\mathrm{H} \delta: 7.096, \mathrm{H \varepsilon}: 10.111,7.539, \\
\mathrm{H} \zeta: 7.355,7.021, \mathrm{H \eta}: 7.103\end{array}$ \\
\hline
\end{tabular}




\section{Table 3 (on next page)}

${ }^{1} \mathrm{H}$ and ${ }^{13} \mathrm{C}$ chemical shifts of Locmi-AKH-I.

${ }^{1} \mathrm{H}$ and ${ }^{13} \mathrm{C}$ chemical shifts of Locmi-AKH-I in DPC micelle solution at $285 \mathrm{~K}$, a pH of 5.0 and 20 $\mathrm{mM}$ phosphate buffer. 
1 Table 3 Locmi-AKH-I; pELNTPNWGT-NH2 in DPC solution $\mathrm{pH} 5.0$, Temp $=285 \mathrm{~K}$

2

\begin{tabular}{|c|c|c|c|c|c|}
\hline \# & Res & $\mathbf{H N}$ & $H_{\alpha}\left(C_{\alpha}\right)$ & $\mathbf{H}_{\beta}\left(H_{\beta^{\prime}}\right)\left(C_{\beta}\right)$ & Others \\
\hline 1 & Glu & & 4.09 & 1.642 & $\mathrm{H} \gamma: 1.541,1.145$ \\
\hline \multirow[t]{2}{*}{2} & Leu & 8.271 & $4.32(54.7)$ & $1.501,1.715$ & $\mathrm{H} \gamma: 1.620, \mathrm{H} \delta: 0.978,0.964$ \\
\hline & & & & $(41.8)$ & $\mathrm{C} \delta 123.4, \mathrm{C} \delta 225.0$ \\
\hline \multirow[t]{2}{*}{3} & Asn & 8.491 & 4.71 & $2.715,2.806$ & $\mathrm{H} \delta: 6.871,7.585$ \\
\hline & & & & $(38.6)$ & \\
\hline \multirow[t]{2}{*}{4} & Phe & 7.768 & $4.67(52.3)$ & $2.984,3.072$ & $\mathrm{H} \delta: 7.182, \mathrm{H} \varepsilon: 7.260, \mathrm{H} \zeta: 7.289$ \\
\hline & & & & $(40.3)$ & 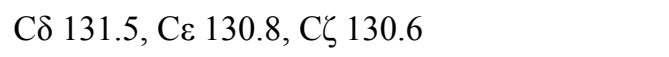 \\
\hline \multirow[t]{2}{*}{5} & Thr & 7.987 & $4.49(57.8)$ & 4.015 & $\mathrm{H} \gamma: 1.080$ \\
\hline & & & & $(69.9)$ & $\mathrm{C} \gamma 220.7$ \\
\hline \multirow[t]{2}{*}{6} & Pro & & $4.44(59.0)$ & $2.031,2.586$ & $\mathrm{H} \gamma: 2.418$ \\
\hline & & & & $(27.8)$ & $\mathrm{C} \gamma 31.9$ \\
\hline \multirow[t]{2}{*}{7} & Asn & 8.336 & 4.68 & $2.672,2.801$ & $\mathrm{H} \delta: 6.851,7.563$ \\
\hline & & & & $(35.6)$ & \\
\hline \multirow[t]{2}{*}{8} & $\operatorname{Trp}$ & 7.529 & 4.74 & 3.301 & 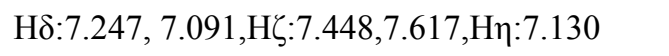 \\
\hline & & & & $(29.7)$ & 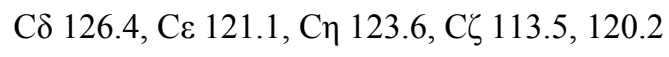 \\
\hline 9 & Gly & 8.316 & $3.94,4.05(45.3)$ & & \\
\hline 10 & Thr & 7.988 & $4.288(61.5)$ & & $\mathrm{H} \gamma: 1.207 \mathrm{C} \gamma 21.3$ \\
\hline
\end{tabular}

3

4 


\section{Table 4 (on next page)}

${ }^{1} \mathrm{H}$ chemical shifts of Aedae-AKH.

${ }^{1} \mathrm{H}$ chemical shifts of Aedae-AKH in DPC micelle solution at $285 \mathrm{~K}$, a pH of 5.0 and $20 \mathrm{mM}$ phosphate buffer. 
1 Table 4 Aedae-AKH-I in DPC solution $\mathrm{pH}$ 5.0, Temp $=285 \mathrm{~K}$

$\begin{array}{llllll}\# & \text { Res } & \text { HN } & \text { HA } & \text { HB }\left(\mathbf{H B}^{\prime}\right) & \text { Others } \\ 1 & \text { Glu } & & 4.41 & 2.36 & \mathrm{H} \gamma: 2.53,1.962 \\ 2 & \text { Leu } & 8.26 & 4.38 & 1.47,1.66 & \mathrm{H} \gamma: 1.68, \mathrm{H} \delta: 0.91,0.932 \\ 3 & \text { Thr } & 8.20 & 4.33 & 4.18 & \mathrm{H} \gamma: 1.10 \\ 4 & \text { Phe } & 7.82 & 4.66 & 3.28 & \mathrm{H} \delta: 7.15, \mathrm{H} \varepsilon: 7.22, \mathrm{HZ}: 7.25 \\ 5 & \text { Thr } & 7.96 & 4.42 & 3.93 & \mathrm{H} \gamma: 1.02 \\ 6 & \text { Pro } & & 4.42 & 1.44,1.56 & \mathrm{H} \gamma: 1.97,2.53, \mathrm{H} \delta: 3.08,3.38 \\ 7 & \text { Ser } & 8.28 & 4.25 & 3.81 & \\ 8 & \text { Trp } & 7.82 & 4.63 & 2.93,3.04 & \mathrm{H} \delta: 7.21, \mathrm{H} \varepsilon: 9.99,7.06, \mathrm{H} \zeta: 7.40,7.58, \mathrm{H \eta}: 7.09\end{array}$

2

3 


\section{Table 5 (on next page)}

List of interactions between peptide and receptor in the binding pocket of Schgr-AKHR. 
1 Table 5. List of interactions of ligand/receptor in binding pocket of Schgr-AKHR receptor.

\begin{tabular}{|l|l|l|l|l|l|}
\hline \multicolumn{2}{|c|}{ Schgr_AKH } & \multicolumn{2}{c|}{ Locmi-AKH } & \multicolumn{2}{c|}{ Aedae-AKH } \\
\hline Ligand & Receptor & Ligand & Receptor & Ligand & Receptor \\
\hline $\mathrm{pE}^{1} \mathrm{O}_{\varepsilon 1}$ & His169 & $\mathrm{pE}^{1} \mathrm{CO}$ & Lys281 & $\mathrm{pE}^{1} \mathrm{CO}$ & His169 \\
\hline $\mathrm{N}^{3} \mathrm{NH}_{2}$ & Cys269 & $\mathrm{N}^{3} \mathrm{CO}$ & Trp273 & $\mathrm{pE}^{1} \mathrm{O}_{\varepsilon 1}$ & Lys281 \\
\hline $\mathrm{F}^{4} \pi-\pi$ stack & Trp93 & $\mathrm{T}^{5} \mathrm{OH}$ & $\mathrm{Gln} 287$ & $\mathrm{~S}^{7} \mathrm{OH}$ & Cys178 \\
\hline $\mathrm{S}^{5} \mathrm{OH}$ & Ser92 & $\mathrm{N}^{7} \mathrm{CO}$ & Trp87 & $\mathrm{S}^{7} \mathrm{NH}$ & Cys178 \\
\hline $\mathrm{G}^{7} \mathrm{NH}$ & Lys288 & $\mathrm{N}^{7} \mathrm{NH}_{2}$ & Ser92 & $\mathrm{W}^{8} \mathrm{H}_{\varepsilon 1}$ & Ser180 \\
\hline $\mathrm{G}^{7} \mathrm{CO}$ & Trp87 & $\mathrm{T}^{10} \mathrm{CO}$ & Lys288 & $\mathrm{W}^{8} \mathrm{NH}_{2}$ & Ser183 \\
\hline $\mathrm{W}^{8} \mathrm{CO}$ & Lys288 & $\mathrm{W}^{8} \pi-\pi$ stack & His169 & & \\
\hline $\mathrm{W}^{8} \pi-\pi$ stack & Tyr175 & $\mathrm{T}^{10} \mathrm{NH}_{2}$ & His28 & & \\
\hline & & & & & \\
\hline
\end{tabular}

2 